
\title{
$\begin{array}{ll}\text { Research Square } & \begin{array}{l}\text { Preprints are preliminary reports that have not undergone peer review. } \\ \text { They should not be considered conclusive, used to inform clinical practice, } \\ \text { or referenced by the media as validated information. }\end{array}\end{array}$
}

\section{A Multi-Analytical Study of the Palette of Impressionist and Post- Impressionist Puerto Rican Artists}

\author{
Marc Vermeulen \\ Northwestern University / Art Institute of Chicago Center for Scientific Studies in the Arts (NU-ACCESS) \\ Annette S. Ortiz Miranda \\ Northwestern University / Art Institute of Chicago Center for Scientific Studies in the Arts (NU-ACCESS) \\ Diego Tamburini \\ The British Museum \\ Sol Rivera \\ Museo de Arte de Puerto Rico \\ Marc Walton ( $\sim$ marc.walton@northwestern.edu ) \\ Northwestern University / Art Institute of Chicago Center for Scientific Studies in the Arts (NU-ACCESS)
}

\section{Research Article}

Keywords: Caribbean art, MA-XRF, Reflectance Imaging Spectroscopy, HPLC-DAD-MS/MS, Synthetic Organic Pigments, Francisco Oller

Posted Date: February 7th, 2022

DOI: https://doi.org/10.21203/rs.3.rs-1322547/v1

License: (a) (i) This work is licensed under a Creative Commons Attribution 4.0 International License. Read Full License

Version of Record: A version of this preprint was published at Heritage Science on April 4th, 2022. See the published version at https://doi.org/10.1186/s40494-022-00683-9. 


\section{Abstract}

This paper presents the pigment characterization in six impressionist and post-impressionist paintings by three leading Puerto Rican artists: Francisco Oller (1833 - 1917), José Cuchí y Arnau (1857-1925), and Ramón Frade (1875-1954). The paintings, belonging to the Museo de Arte de Puerto Rico (San Juan, Puerto Rico), were investigated through a combination of non- and micro-invasive scientific techniques. The noninvasive application of macro-X-ray fluorescence (MA-XRF) and reflectance imaging spectroscopy (RIS) to the characterization of Puerto Rican artists' palette is presented for the first time. The non-invasive approach was integrated with spectroscopic techniques such as Raman and/or Fourier-transform infrared (FTIR) spectroscopy, as well as high pressure liquid chromatography coupled to diode array detector and tandem mass spectrometry (HPLC-DAD-MS/MS), when sampling was possible. While this technical investigation reveals pigments that are typical for late 19th/early 20th century paintings, it also emphasizes some unexpected findings, including the use of cobalt green and synthetic yellow lakes, which enabled the date attributed to some of the paintings to be refined to post 1910 rather than their current dates of ca. 1890. This study confirms that the Puerto Rican artist's palettes are very similar to their European contemporaries, underscoring both their European training and their attempt to adapt these methods of painting to a new Caribbean identity emerging from the Spanish American War.

\section{Introduction}

When one thinks of impressionist and post-impressionist painters (Monet, Renoir, Pissarro, and Cezanne, to only cite a few), Puerto Rican artists may not be the first that come to mind. However, Puerto Rico - a Spanish colony until 1898 - produced several late-19th $/$ early $20^{\text {th }}$ century masters, including Francisco Oller (1833 - 1917) who became known as the first Latin American painter to adopt the impressionists' artistic vocabulary of light and color to document the Puerto Rican landscape. His paintings of sugarcane plantations and still-lives of tropical fruits are eagerly sought by private collectors and found in many major museums around the world, including the Musée d'Orsay, the Brooklyn Museum, the Museo del Barrio, and several cultural institutions in Puerto Rico (Museo de Arte de Ponce, Instituto de Cultura Puertorriqueña and the Museo de Arte de Puerto Rico) [1]. While he had initial art training in San Juan, he went abroad for further study in Spain and France, where he painted with Monet and even introduced Camille Pissarro, another Caribbean native from the Danish West Indies, to Paul Cézanne [1]. Unlike Pissarro, however, Oller chose not to stay in Europe and instead settled in Puerto Rico where he remained until his death in 1917. Oller's artistic influence was significant and today he is recognized as one of the foremost artists to express a Caribbean visual culture and identity [1].

Oller's work depicting Puerto Rico also influenced many other Puerto Rican artists, such as José Cuchí y Arnau (1857 - 1925) and Ramón Frade (1875 - 1954). Many from this next generation of Puerto Rican artists followed in Oller's footsteps and left the island to be trained abroad. Notably, in 1874, José Cuchí y Arnau moved to Barcelona where he was known for his portraits representing popular characters of Spanish culture [2]. In contrast, Ramón Frade was trained in the Dominican Republic under the supervision of Spanish and French teachers becoming a major champion for producing artistic works that helped establish a Puerto Rican identity [3]. Further emphasizing their embrace of the European culture, Cuchí y Arnau was awarded a gold medal at the Paris Exposition Universelle in 1900 while Frade exhibited his works at the Paris Salon that same year.

Through their ties with Puerto Rico and the Caribbean in general, these artists helped transform painting in the Caribbean with a vibrant exchange of ideas, materials, and the latest technical developments in early European paintings. In the early 1900, European artistry started shifting toward more avant garde movements such as fauvism, expressionism, and cubism [4,5]. Yet, these Puerto Rican artists chose to hold on to a more representational style to appeal to a Caribbean-centered audience. While the color palette and technique of French and American impressionist and post-impressionist artists have been extensively studied [6-11], Puerto Rican artists have not attained the same level of appreciation as their European contemporaries, reached the same broad audiences, nor received the same scientific attention. Consequently, little is known about their use of materials and painting techniques.

To redress this gap in our knowledge about the painting methods of the 'Caribbean impressionists,' this investigation considers six paintings by Francisco Oller, José Cuchí y Arnau and Ramón Frade. Primarily MA-XRF and reflectance imaging spectroscopy (RIS) are used here to identify pigments and map their distribution in paintings based on the pigments' specific elemental composition and molecular response [12-16]. For example, vermilion (HgS), which is the only (red) artist pigment containing $\mathrm{Hg}$, and for which the reflectance spectrum has a specific inflection point around $590 \mathrm{~nm}$, is easily identified through its elemental and molecular characterization [17]. On the other hand, pigments such as Emerald and/or Scheele's greens have a very specific elemental composition ( $\mathrm{Cu}$ and As) as determined by their XRF responses, yet can have a reflectance spectrum similar to a mixture of Prussian blue and a yellow pigment such as chrome yellow [18,19]. While usually not identified solely by MA-XRF [20], Prussian blue and ultramarine blue have unique reflectance spectra, which allow for their unambiguous identification. Apart from yellow ochres, which have a very specific reflectance spectrum [17], yellow colorants, in general, are difficult to identify through RIS. However, MA-XRF is often considered specific enough that it becomes possible to associate elemental co-presence with a specific yellow pigment, e.g. $\mathrm{Pb} / \mathrm{Sn}$ being a strong indication for lead tin yellow; $\mathrm{Pb} / \mathrm{Cr}$ pointing towards chrome yellow; $\mathrm{Pb} / \mathrm{Sb}$ for $\mathrm{Naples}$ yellow; $\mathrm{Ba} / \mathrm{Cr}$ indicative of lemon yellow, etc. Therefore, as shown by our examples here, by coupling both techniques, it becomes easier to differentiate between various pigments or mixtures of pigments for a given color, based on the combination of the color analyzed, its elemental composition

Page $2 / 24$ 
and reflectance spectrum. Nevertheless, MA-XRF only provides elemental composition and the electronic transitions associated with RIS typically produce a broad and sometimes unspecific molecular response which means that additional spectroscopic methods are sometimes needed for an unambiguous pigment identification. For this study we also employed Raman and FTIR as well as scanning electron microscopy coupled to energy dispersive X-ray spectrometry (SEM-EDX) [16,21]. In the case of organic colorants, chromatographic techniques, such as high pressure liquid chromatography (HPLC) coupled to diode array detector (DAD) and/or mass spectrometry (MS) applied to micro-samples were integrated into the analytical protocol [22].

Following this rationale, this study represents an opportunity to investigate the material influence of various cultures (European, American, and Caribbean) on the late $19^{\text {th }}$ century artistic production in Latin America through the determination of the artists' palette. While scholarship on the philosophical and art historical perspectives of Latin American and Caribbean artists from the $19^{\text {th }}$ century is already robust [23-31], no studies have been conducted to address materials comprising the Puerto Rican artistic palette. This study sets a foundation for further studies focusing on Caribbean and Central American art.

\section{Materials And Methods}

\section{Painting $\underline{s}$}

Figure 1 presents the six paintings by Puerto Rican artists belonging to the permanent collection of the Museo de Arte de Puerto Rico (San Juan, Puerto Rico) that were analyzed during this study. According to biographical and historical data, all six paintings were likely to be realized between 1890 and 1905, a time of important political and social changes in Puerto Rico. Table 1 summarizes all information associated with the six paintings studied.

When possible, paint microsamples were removed with a scalpel from selected locations of the paintings, mostly on the edges, to investigate the ground composition, the layer build-up and some of the pigments used. The localization of the various samples is indicated by the red circles in Figure 1.

Some samples were mounted as cross-sections (CS1 to CS5) prior to be analyzed. The cross-sections were prepared by embedding the specimens in Buehler Epo Thin 2 epoxy. The cured cross-sections were polished under a flow of water with Buehler polishing cloths with a sequence of grits from 500 to 2400 grade until the fragment surface was nearly exposed. Dry polishing was continued using a $1 \mu$ m diamond suspension (Buehler MetaDi) to expose the samples and obtain a mirror-like surface. The paint cross sections were rinsed in hexane to reduce resin contaminations.

\section{MA-XRF}

The MA-XRF scanner developed at NU-ACCESS [32] was used. The instrument is equipped with a transmission $12 \mathrm{~W}$ Rh anode X-Ray tube (MOXTEK Inc., Orem, UT) coupled to a removable polycapillary optic (XOS Inc, East Greenbush, NY) yielding a beam diameter of $100 \mu \mathrm{m}$ at a $4.8 \mathrm{~cm}$ focal point. Two laser pointers, mounted in such a way that their intersection point coincides with the cross-point of the incident $X$-ray beam and detector axis, allow for optimizing both excitation and detection conditions. X-rays are detected by means of a $50 \mathrm{~mm}^{2}$ active collimated area Silicon Drift Detector (SDD, Bruker Nano Analytics), equipped with a CUBE preamplifier associated with DANTE digital pulse processor (DANTE DPP, Bruker Nano Analytics), with an energy resolution of $132 \mathrm{eV}$ at the Mn Ka line (5.9 keV). The instrument was operated at $30 \mathrm{kV}$ and $400 \mu \mathrm{A}$. The elemental 2D mapping of the objects surface was achieved through an automatic XY raster scanning with varying per pixel acquisition times and step size. The detailed per pixel acquisition times and step size used for each painting are given in Table S1. The elemental maps were produced using PyMCA software [33]. In the case when several maps were required to scan the entire painting, the various maps were stitched together using registration and stitching plugins available in the open-source image processing package Fiji suite [34,35].

Table 1. Summary of the analyzed paintings 


\begin{tabular}{|c|c|c|c|c|c|c|}
\hline Painting's original titles & Artist & $\begin{array}{l}\text { Year of } \\
\text { creation }\end{array}$ & Dimension & Medium & $\begin{array}{l}\text { Object } \\
\text { number }\end{array}$ & $\begin{array}{l}\text { Cross } \\
\text { section/Samples }\end{array}$ \\
\hline Trapiche meladero & Francisco Oller & 1890 & $\begin{array}{l}43.8 \times 52.1 \\
\mathrm{~cm}\end{array}$ & $\begin{array}{l}\text { Oil on } \\
\text { canvas }\end{array}$ & Trapiche & CS1 \\
\hline \multirow[t]{2}{*}{ Bodegón con guanábanas } & & \multirow[t]{2}{*}{ Circa 1890} & \multirow[t]{2}{*}{$52.1 \times 80 \mathrm{~cm}$} & \multirow{2}{*}{$\begin{array}{l}\text { Oil on } \\
\text { wood }\end{array}$} & \multirow[t]{2}{*}{ CAM-1329 } & SCR1 \\
\hline & & & & & & SCR2 \\
\hline \multirow[t]{2}{*}{$\begin{array}{l}\text { Bodegón con aguacates y } \\
\text { utensilios }\end{array}$} & & \multirow[t]{2}{*}{$1890-91$} & \multirow[t]{2}{*}{$\begin{array}{l}52.4 \times 83.8 \\
\mathrm{~cm}\end{array}$} & \multirow[t]{2}{*}{$\begin{array}{l}\text { Oil on } \\
\text { canvas }\end{array}$} & \multirow[t]{2}{*}{ CAM-1328 } & \\
\hline & & & & & & SCR3 \\
\hline La Chula & $\begin{array}{l}\text { José Cuchí y } \\
\text { Arnau }\end{array}$ & 1895 & $\begin{array}{l}68.6 \times 109.2 \\
\mathrm{~cm}\end{array}$ & $\begin{array}{l}\text { Oil on } \\
\text { canvas }\end{array}$ & $2004-005$ & CS3 \\
\hline Mujer en la playa & & 1897 & $\begin{array}{l}49.5 \times 34.9 \\
\mathrm{~cm}\end{array}$ & $\begin{array}{l}\text { Oil on } \\
\text { canvas }\end{array}$ & $2004-007$ & CS4 \\
\hline Rêverie d'amour & Ramón Frade & 1905 & $35.9 \times 61 \mathrm{~cm}$ & $\begin{array}{l}\text { Oil on } \\
\text { wood }\end{array}$ & $2003-05-02$ & CS5 \\
\hline
\end{tabular}

\section{Reflectance Imaging Spectroscopy_(RIS).}

RIS data were acquired using a Resonon Pika II Pushbroom system (Resonon, Inc., Bozeman, MT, USA) in the 400-900 nm range with spectral resolution of $2.1 \mathrm{~nm}$, with a total of 240 channels. The system was connected to a stage allowing the scanning of about $30 \mathrm{~cm}$ of the object's width, with an integration time of 1 second, and a spatial per-pixel resolution of $0.3 \times 0.3 \mathrm{~mm}$ or $0.5 \times 0.5 \mathrm{~mm}$ depending on the distance between the painting and the camera's objective (which was changed according to the paintings dimensions to accommodate the access time to the paintings). The spatial resolution and varying number of cubes acquired for each painting are indicated in Table S1. During acquisition, the object was illuminated using two broad spectrum tungsten halogen lamps placed at $45^{\circ}$ of the objects normal. A Spectralon diffuse white reflectance standard (Labsphere, North Sutton, USA) was used as a calibration target to convert the image cubes to diffuse reflectance. Hyperspectral acquisition was performed using the SpectrononPro software (Resonon, Inc., Bozeman, MT, USA). The raw hyperspectral data cubes were converted to a tiff stack in Fiji and the partially overlapped areas of two cubes from the same painting were registered and stitched together using the open-source image processing package Fiji suite [34,35], prior to further processing. The final complete cube and its RGB image were registered to MA-XRF maps for comparison purposes.

Spectral stacks were further processed using our previously described software pipeline [36] that utilizes UMAP, density cluster mapping, and non-negative least square fitting procedure to extract the various pigments spectra (endmembers) and recreate their distribution maps. The data processing was performed in a Jupyter Notebook running in Python. The cosine distance metric was used for the data reduction and a number of neighbors of 10 was chosen by default for the density clustering as described by our protocol [36]. The threshold and minimum distance used for the $2 \mathrm{~d}$ histogram were tailored to each painting and are given in the associated figure legends.

Comparison of the reflectance spectra with the in-house, published, and online databases $[17,22,37,38]$ was used to tentatively identify the pigments employed. Pigment possibilities were also narrowed-down with the help of the elemental distribution maps obtained using MA-XRF.

\section{SEM-EDX}

Backscattered electron (BSE) images and elemental distribution maps of cross sections were acquired with a Hitachi S-3400N-II SEM (Hitachi, Tokyo, Japan) equipped with a INCAx-act EDS detection system (Oxford Instruments, Abingdon, United Kingdom) all operated under variable pressure vacuum $(60 \mathrm{~Pa})$. Images and elemental maps were recorded using an acceleration voltage of $20 \mathrm{kV}$ and a $90-\mathrm{mA}$ probe current. EDS analyses were performed with a working distance of $10 \mathrm{~mm}$ and a minimum of 300000 counts/spectrum. Data was collected and processed using the AZtec software system, v. 3.3 (Oxford Instruments, Abingdon, United Kingdom).

\section{Raman spectroscopy}

Spectra were acquired with a HORIBA LabRAM HR Evolution Confocal Raman spectrometer equipped with an Olympus 50x long working distance objective and a charge-coupled device (CCD) detector. A continuous wave diode laser (Laser Quantum) and a solid-state laser (Horiba Scientific), emitting light at 532 and $785 \mathrm{~nm}$ respectively, were used as the excitation sources and a $600 \mathrm{ruling} / \mathrm{mm}$ holographic grating provided a spectral resolution of $3.2 \mathrm{~cm}^{-1}$. The output laser power was kept below $25 \mathrm{~mW}$, while the number of scans and integration time were adjusted to prevent damage from overheating and according to the spectral response of the samples examined. All spectra were acquired using the HORIBA LabSpec 6 Spectroscopy Suite Software. 
Two micro-samples (approximately $50 \mu \mathrm{g}$ ) taken from CAM-1328 and CAM-1329 (SCR3 and SCR2 respectively) were analyzed by high pressure liquid chromatography coupled to diode array detector and electrospray ionization followed by quadrupole and time of flight detection (HPLCDAD-ESI-Q-ToF). The molecular extraction was performed using a method published in [22], which briefly consists of a double mild extraction procedure, using DMSO first, and secondly a mixture of methanol/acetone/water/0.5M oxalic acid 30:30:40:1 (v/v/v/v).

Analyses were carried out using a 1260 Infinity HPLC (Agilent Technologies), coupled to a 1100 DAD detector (Hewlett-Packard) and to a Quadrupole-Time of Flight tandem mass spectrometer 6530 Infinity Q-ToF detector (Agilent Technologies) by a Jet Stream ESI interface (Agilent Technologies). Separation was achieved using a Zorbax Extend-C18 column (2.1 mm $\times 50 \mathrm{~mm}, 1.8 \mu \mathrm{m}$ particle size) with a $0.4 \mathrm{~mL} / \mathrm{min}$ flow rate and $40^{\circ} \mathrm{C}$ column temperature, and a gradient of water with $0.1 \%$ formic acid (eluent A) and acetonitrile with $0.1 \%$ formic acid (eluent $B$ ). The elution gradient was programmed as follows: initial conditions $95 \%$ A, followed by a linear gradient to $100 \%$ B in 10 min, and held for 2 min. Re-equilibration time for each analysis was $10 \mathrm{~min} .10 \mu \mathrm{L}$ injection volume was adopted for MS experiments and $20 \mu \mathrm{L}$ for MS/MS experiments.

The DAD detector (cell volume $50 \mu \mathrm{L}$ ) scanned in the range $200-700 \mathrm{~nm}$ with $2 \mathrm{~nm}$ resolution. The ESI operating conditions were: drying gas $\left(\mathrm{N}_{2}\right.$, purity $\left.>98 \%\right)$ temperature $350^{\circ} \mathrm{C}$ and $10 \mathrm{~L} / \mathrm{min}$ flow; capillary voltage $4.0 \mathrm{kV}$; nebulizer gas pressure 40 psig; sheath gas $\left(\mathrm{N}_{2}\right.$, purity $\left.>98 \%\right)$ temperature $375^{\circ} \mathrm{C}$ and flow $11 \mathrm{~L} / \mathrm{min}$. High resolution MS and MS/MS spectra were acquired in both negative and positive mode in the range 100-1700 m/z. The fragmentor was kept at $100 \mathrm{~V}$, nozzle voltage $1000 \mathrm{~V}$, skimmer $65 \mathrm{~V}$, octapole RF $750 \mathrm{~V}$. For the MS/MS experiments, different voltages (from 10 to $40 \mathrm{~V}$ ) in the collision cell were tested for Collision Induced Dissociation (CID), in order to maximize the information obtained from the fragmentation of the single molecules. The collision gas was $\mathrm{N}_{2}$ (purity $99.999 \%$ ). The data were collected by targeted MS/MS acquisition with an MS scan rate of 1.0 spectra/sec and a MS/MS scan rate of 1.0 spectra/sec. Auto-calibration was performed daily using Agilent tuning mix HP0321 (Agilent Technologies) prepared in 90\% water-10\% acetonitrile.

MassHunter Workstation Software was used to carry out diode array detector and mass spectrometer control, data acquisition, and data analysis. In particular, extract ion chromatograms were obtained using the software EIC function and selecting the mass range corresponding to the calculated mass $\pm 0.01 \mathrm{~m} / \mathrm{z}$.

\section{Results}

Non-invasive imaging techniques: MA-XRF and RIS

The color scheme of the paintings studied is rather simple and incorporates mostly shades of white, blue, green, red, yellow, and brown. The MA-XRF distribution maps, RIS endmember maps and associated reflectance curves for CAM-1329 are presented in Fig. 2 and Fig. 3 , respectively.

The XRF maps of $\mathrm{Pb}$ (Fig. 2-Pb) suggest a lead-based ground, likely to be lead white (lead carbonate and/or basic lead carbonate), due to Pb being found throughout the compositions. Further indications of the presence of $\mathrm{Pb}$ in the lower layers are given by the shielding effect of overlying heavy elements, such as $\mathrm{Co}, \mathrm{Zn}, \mathrm{Cr}$, and $\mathrm{Cu}$, which serve to block the $\mathrm{Pb}$ signal found underneath, as well as small areas of loss where $\mathrm{Ca}, \mathrm{Fe}, \mathrm{Ti}, \mathrm{Ba}, \mathrm{Ti}$, and $\mathrm{K}$ - most likely used during previous conservation treatments - are found. The large ochre-based conservation intervention in the area of loss in the plate is further confirmed using RIS (Fig. 3-EM1).

The presence of $\mathrm{Hg}$ in the redder areas such as the brown of the table or the edges of the plate (Fig. 2-Hg) suggests the use of vermilion (mercury sulfide), the sole pigment containing mercury. The use of vermilion is further suggested by RIS, with the characteristic inflection point at $585 \mathrm{~nm}$ (Fig. 3-EM6). However, the complex curve observed with absorbances at 455, 560, and 640 nm may also suggest the use of an oxide/oxy - hydroxide-based ochre mixed with the vermilion.

XRF maps also imply the presence of a Cr-based green in most green areas of the composition, including the guanábanas (Fig. 2-Cr). The Crbased pigment is likely to be viridian (hydrated chromium oxide), chrome(III)oxide green and/or a mixture of chrome yellow and blue pigments. The spectral features, and specifically the shoulder at $705 \mathrm{~nm}$ observed in RIS (Fig. 3-EM3-5), tend to indicate the use of viridian or chrome(III)oxide green, rather than a mixture of chrome yellow and blue pigments. However, neither MA-XRF nor RIS allows for their differentiation, hence Raman spectroscopy was applied (section "Micro-invasive investigation: optical microscopy, SEM-EDX, and Raman spectroscopy").

The Cr-based green does not appear to be the sole green pigment used in the painting. The co-occurrence of As and Cu also suggests that Oller used Emerald green (copper acetoarsenite) and/or Scheele's green (copper arsenite, Fig. 2-As/Cu) in the green highlights of the fruits. The presence of the emerald and/or Scheele's green was also confirmed by RIS through the inflection point and maximum absorbance at 505 and $670 \mathrm{~nm}$, respectively (Fig. 3-EM7). 
Despite not being identified by MA-XRF due to its constituting elements being too light to be picked up by the technique, RIS highlighted the use of ultramarine blue (sulfur-containing sodium aluminum silicate) in the blue highlights (Fig. 3-EM2). Co, an element often associated with cobalt blue (cobalt(II) oxide-aluminum oxide), was identified by MA-XRF in the green areas of the guanábanas (Fig. 2-Co). The Co was observed in association with Zn (Fig. 2-Zn), which may suggest a combined used of cobalt blue and zinc white (zinc oxide). However, this association would prove peculiar due to the rather dark color of the depicted fruit, for which zinc white would not be expected. Furthermore, the co-presence of Co and Zn may also be associated with a cobalt green, a cobalt oxide-doped zinc oxide pigment. Even though it was not possible to confirm the presence of the pigment using minimally invasive techniques due to the lack of sample in the area, cobalt green was identified with Raman spectroscopy in the light blue sky of Trapiche Meladero (see section "Micro-invasive investigation: optical microscopy, SEM-EDX, and Raman spectroscopy"), another of Oller's painting for which Co and Zn were identified using MA-XRF (Fig. S1). This finding may support the possible use of the pigment in this second painting by the same artist.

The presence of $\mathrm{Zn}$ and $\mathrm{Cr}$ in the guanábanas could also be associated with the use of yellow zinc chromate. However, both elements do not seem to overlap contrary to Co and Zn as suggested by the RGB composite images presented in Fig. 4, and therefore, supporting further the use of cobalt green and/or a mixture of cobalt blue and zinc white.

Except for the warm background, yellow tones are only sparsely used throughout the composition. Yellow is mostly observed in the yellow knife handle and in the light-yellow filling of the bowl. However, neither these elements nor the background yields any elemental response that could be linked to yellow pigments, such as $\mathrm{Cr}$ (for chrome or lemon yellows), often identified in paintings from impressionist and post-impressionist periods. RIS, while not able to identify the pigment used, did however set apart both areas (Fig. 3-EM8 and Fig. 3-EM9 for the knife handle and background, respectively), with features at 565 and $650 \mathrm{~nm}$ possibly associated with an ochre pigment, not identified through the presence of Fe in MA-XRF. The lack of elemental response in the yellow areas may suggest the use of organic lakes and/or synthetic organic pigments, further suggested by the absorbance band at $440 \mathrm{~nm}$ observed in RIS (Fig. 3-EM8 and Fig. 3-EM9). Nonetheless, non-invasive imaging techniques do not enable most yellow colorants to be identified and further analyses more suited for the identification of organic pigments such as Raman spectroscopy or LCMS are necessary (section "Micro-invasive chromatographic analysis: HPLC-DAD-MS/MS").

Only MA-XRF and RIS data associated with CAM-1329 are presented here. However, a similar train of thoughts applied to the distribution maps (MA-XRF and RIS) of the five other paintings by Oller, Cuchí y Arnau and Frade was followed. The corresponding results are shown in Supplementary Information (Fig. S1 to Fig. S10). A summary of the results and tentative pigment identifications obtained from the non-invasive imaging techniques are present in Table 2 for all paintings under investigation.

Table 2. Overview of the materials identified or tentatively identified through non-invasive analysis of the six paintings investigated. 


\begin{tabular}{|c|c|c|c|c|c|c|c|c|}
\hline \multirow[t]{3}{*}{ Painting } & \multirow[t]{3}{*}{ Color } & \multicolumn{3}{|c|}{ MA-XRF/SEM-EDX } & \multicolumn{4}{|c|}{ Reflectance spectroscopy/ $\mu$-Reflectance spectroscopy } \\
\hline & & Element & Figure/ & $\begin{array}{l}\text { Tentative } \\
\text { pigment }\end{array}$ & $\begin{array}{l}\text { Distribution } \\
\text { map / }\end{array}$ & $\begin{array}{l}\text { Maximum } \\
\text { absorption }\end{array}$ & $\begin{array}{l}\text { Inflection } \\
\text { point }\end{array}$ & $\begin{array}{l}\text { Tentative pigment } \\
\text { identification }\end{array}$ \\
\hline & & & $\begin{array}{l}\text { Distribution } \\
\text { map }\end{array}$ & identification & & & & \\
\hline \multirow[t]{8}{*}{ Trapiche } & Green & $\mathrm{Cr}$ & Fig. S1-Cr & $\begin{array}{l}\text { Viridian, } \\
\text { chrome(III)oxide } \\
\text { green and/or } \\
\text { chrome yellow } \\
+ \text { blue }\end{array}$ & $\begin{array}{l}\text { Fig. S2- } \\
\text { EM2 }\end{array}$ & $\begin{array}{l}450,600 \\
705\end{array}$ & 745 & $\begin{array}{l}\text { Viridian and/or } \\
\text { chrome(III)oxide green }\end{array}$ \\
\hline & Blue & Co & Fig. S1-Co & Cobalt blue & $\begin{array}{l}\text { Fig. S2- } \\
\text { EM1 }\end{array}$ & $\begin{array}{l}540,580 \\
630\end{array}$ & 680 & Cobalt blue \\
\hline & Red & $\mathrm{Hg}$ & Fig. S1-Hg & Vermilion & $\mathrm{N} / \mathrm{A}$ & $\mathrm{N} / \mathrm{A}$ & $\mathrm{N} / \mathrm{A}$ & $\mathrm{N} / \mathrm{A}$ \\
\hline & Yellow & $\mathrm{Fe}$ & Fig. S1-Fe & $\begin{array}{l}\text { Iron oxide/oxy- } \\
\text { hydroxide }\end{array}$ & $\begin{array}{l}\text { Fig. S2- } \\
\text { EM4 }\end{array}$ & 630,900 & $\mathrm{~N} / \mathrm{A}$ & Ochre \\
\hline & Brown & & & & $\begin{array}{l}\text { Fig. S2- } \\
\text { EM3 }\end{array}$ & & & \\
\hline & \multirow{2}{*}{$\begin{array}{l}\text { Dark / } \\
\text { black }\end{array}$} & \multirow[t]{2}{*}{ Co, $\mathrm{Ca}$} & Fig. S1-Co & Cobalt blue & \multirow{2}{*}{$\begin{array}{l}\text { Fig. S2- } \\
\text { EM5 }\end{array}$} & \multirow[t]{2}{*}{580,630} & \multirow[t]{2}{*}{680} & \multirow[t]{2}{*}{ Cobalt blue } \\
\hline & & & Fig. S1-Ca & Bone black & & & & \\
\hline & Ground & $\mathrm{Pb}$ & Fig. S1-Pb & Lead white & $\mathrm{N} / \mathrm{A}$ & $\mathrm{N} / \mathrm{A}$ & $\mathrm{N} / \mathrm{A}$ & $\mathrm{N} / \mathrm{A}$ \\
\hline \multirow{18}{*}{$\begin{array}{l}\text { CAM- } \\
1329\end{array}$} & \multirow[t]{7}{*}{ Green } & \multirow{7}{*}{$\begin{array}{l}\mathrm{Cr}, \mathrm{Co} \\
\mathrm{Zn}, \mathrm{As}, \\
\mathrm{Cu}\end{array}$} & \multirow[t]{3}{*}{ Fig. 2-Cr } & \multirow{3}{*}{$\begin{array}{l}\text { Viridian, } \\
\text { chrome(III)oxide } \\
\text { green and/or } \\
\text { chrome yellow } \\
+ \text { blue }\end{array}$} & Fig. 3-EM3 & \multirow[t]{5}{*}{$\begin{array}{l}450,600 \\
705\end{array}$} & \multirow[t]{5}{*}{745} & \multirow{5}{*}{$\begin{array}{l}\text { Viridian/chrome(III)oxide } \\
\text { green + ultramarine blue } \\
\text { Cobalt blue/Cobalt } \\
\text { green } \\
\text { Zinc white/Cobalt green }\end{array}$} \\
\hline & & & & & Fig. 3-EM4 & & & \\
\hline & & & & & \multirow[t]{3}{*}{ Fig. 3-EM5 } & & & \\
\hline & & & Fig. 2-Co & Cobalt blue + & & & & \\
\hline & & & Fig. 2-Zn & $\begin{array}{l}\text { and/or cobalt } \\
\text { green }\end{array}$ & & & & \\
\hline & & & Fig. 2-As & \multirow{2}{*}{$\begin{array}{l}\text { Emerald and/or } \\
\text { Scheele's green }\end{array}$} & Fig. 3-EM7 & \multirow[t]{2}{*}{450,670} & & \multirow[t]{2}{*}{ Emerald green } \\
\hline & & & Fig. 2-Cu & & & & & \\
\hline & Blue & $\mathrm{N} / \mathrm{A}$ & $\mathrm{N} / \mathrm{A}$ & $\mathrm{N} / \mathrm{A}$ & Fig. 3-EM2 & 410,600 & 690 & Ultramarine blue \\
\hline & \multirow[t]{2}{*}{ Yellow } & \multirow[t]{2}{*}{$\mathrm{N} / \mathrm{A}$} & \multirow[t]{2}{*}{$\mathrm{N} / \mathrm{A}$} & \multirow[t]{2}{*}{$\mathrm{N} / \mathrm{A}$} & Fig. 3-EM8 & \multirow{2}{*}{$\begin{array}{l}440,565 \\
650\end{array}$} & \multirow[t]{2}{*}{500,695} & \multirow[t]{2}{*}{ Possibly organic yellow } \\
\hline & & & & & Fig. 3-EM9 & & & \\
\hline & Brown & $\mathrm{Hg}$ & Fig. 2-Hg & Vermilion & Fig. 3-EM6 & $\begin{array}{l}455,560 \\
640\end{array}$ & 580 & Ochre + vermilion \\
\hline & White & $\mathrm{Pb}$ & Fig. 2-Pb & Lead white & $\mathrm{N} / \mathrm{A}$ & $\mathrm{N} / \mathrm{A}$ & $\mathrm{N} / \mathrm{A}$ & $\mathrm{N} / \mathrm{A}$ \\
\hline & Ground & $\mathrm{Pb}$ & Fig. 2-Pb & Lead white & $\mathrm{N} / \mathrm{A}$ & $\mathrm{N} / \mathrm{A}$ & $\mathrm{N} / \mathrm{A}$ & $\mathrm{N} / \mathrm{A}$ \\
\hline & Retouching & $\mathrm{Ca}, \mathrm{Fe}$ & Fig. 2-Ca & Calcium & & & & \\
\hline & & & Fig. 2-Fe & Iron oxide/oxy & Fig. 3-EM1 & 630,900 & & Ochre \\
\hline & & & Fig. 2-Ti & hydroxide & & & & \\
\hline & & & Fig. 2-Ba & Titanium white & & & & \\
\hline & & & & Barium sulfate & & & & \\
\hline
\end{tabular}

Table 2. Overview of the materials identified or tentatively identified through non-invasive analysis of the six paintings investigated. Continued 


\begin{tabular}{|c|c|c|c|c|c|c|c|c|}
\hline \multirow[t]{2}{*}{ Painting } & \multirow[t]{2}{*}{ Color } & \multicolumn{3}{|l|}{ MA-XRF } & \multicolumn{4}{|c|}{ Reflectance spectroscopy } \\
\hline & & Element & $\begin{array}{l}\text { Figure/ } \\
\text { Distribution } \\
\text { map }\end{array}$ & $\begin{array}{l}\text { Tentative pigment } \\
\text { identification }\end{array}$ & $\begin{array}{l}\text { Distribution } \\
\text { map / } \\
\text { Spectrum }\end{array}$ & $\begin{array}{l}\text { Maximum } \\
\text { absorption } \\
(\mathrm{nm})\end{array}$ & $\begin{array}{l}\text { Inflection } \\
\text { point } \\
(\mathrm{nm})\end{array}$ & $\begin{array}{l}\text { Tentative } \\
\text { pigment } \\
\text { identification }\end{array}$ \\
\hline \multirow{19}{*}{$\begin{array}{l}\text { CAM- } \\
1328\end{array}$} & \multirow[t]{3}{*}{ Green } & \multirow{3}{*}{$\begin{array}{l}\mathrm{Cu}, \mathrm{As}, \\
\mathrm{Cr}\end{array}$} & Fig. S3-Cu & Emerald green and/or & \multirow{3}{*}{$\begin{array}{l}\text { Fig. S4- } \\
\text { EM1 }\end{array}$} & \multirow[t]{3}{*}{450,670} & & \multirow[t]{3}{*}{ Emerald green } \\
\hline & & & Fig. S3-As & & & & & \\
\hline & & & Fig. S3-Cr & $\begin{array}{l}\text { Viridian, chrome(III)oxide } \\
\text { green and/or chrome } \\
\text { yellow + blue }\end{array}$ & & & & \\
\hline & Blue & $\mathrm{N} / \mathrm{A}$ & $\mathrm{N} / \mathrm{A}$ & $\mathrm{N} / \mathrm{A}$ & $\begin{array}{l}\text { Fig. S4- } \\
\text { EM5 }\end{array}$ & 590 & 700 & $\begin{array}{l}\text { Ultramarine } \\
\text { blue }\end{array}$ \\
\hline & Red & $\mathrm{Hg}$ & Fig. S3-Hg & Vermilion & $\begin{array}{l}\text { Fig. S4- } \\
\text { EM7 }\end{array}$ & 660 & 590 & $\begin{array}{l}\text { Vermilion- } \\
\text { containing red }\end{array}$ \\
\hline & \multirow[t]{2}{*}{ Yellow } & \multirow[t]{2}{*}{$\mathrm{N} / \mathrm{A}$} & \multirow[t]{2}{*}{$\mathrm{N} / \mathrm{A}$} & \multirow[t]{2}{*}{$\mathrm{N} / \mathrm{A}$} & $\begin{array}{l}\text { Fig. S4- } \\
\text { EM2 }\end{array}$ & 440 & 500 & $\begin{array}{l}\text { Possibly } \\
\text { organic yellow }\end{array}$ \\
\hline & & & & & $\begin{array}{l}\text { Fig. S4- } \\
\text { EM3 }\end{array}$ & & & \\
\hline & \multirow[t]{4}{*}{ Brown } & \multirow{4}{*}{$\begin{array}{l}\mathrm{Fe}, \mathrm{Mn} \\
\mathrm{Hg}, \mathrm{Ca}\end{array}$} & Fig. S3-Fe & Umber & \multirow{4}{*}{$\begin{array}{l}\text { Fig. S4- } \\
\text { EM6 }\end{array}$} & \multirow[t]{4}{*}{$\mathrm{N} / \mathrm{A}$} & \multirow[t]{4}{*}{700} & \multirow{4}{*}{$\begin{array}{l}\text { Unknown } \\
\text { brown, most } \\
\text { likely } \\
\text { containing } \\
\text { ultramarine } \\
\text { blue }\end{array}$} \\
\hline & & & Fig. S3-Mn & & & & & \\
\hline & & & Fig. S3-Hg & Vermilion & & & & \\
\hline & & & Fig. S3-Ca & $\begin{array}{l}\text { Bone black or calcium } \\
\text { carbonate }\end{array}$ & & & & \\
\hline & Ground & $\mathrm{Pb}$ & Fig. S3-Pb & Lead white & N/A & $\mathrm{N} / \mathrm{A}$ & $\mathrm{N} / \mathrm{A}-$ & $\mathrm{N} / \mathrm{A}$ \\
\hline & \multirow[t]{7}{*}{ Retouching } & \multirow[t]{7}{*}{$\begin{array}{l}\mathrm{Ca}, \mathrm{Fe}, \mathrm{K} \\
\mathrm{Ti}, \mathrm{Ba}, \\
\mathrm{Co}, \mathrm{Zn}\end{array}$} & Fig. S3-Fe & $\begin{array}{l}\text { Iron oxide/ } \\
\text { oxy-hydroxide }\end{array}$ & \multirow[t]{7}{*}{$\begin{array}{l}\text { Fig. S4- } \\
\text { EM4 }\end{array}$} & \multirow[t]{7}{*}{630,890} & \multirow[t]{7}{*}{520} & \multirow[t]{7}{*}{ Ochre } \\
\hline & & & Fig. S3-K & Unknown & & & & \\
\hline & & & Fig. S3-Ca & Calcium carbonate & & & & \\
\hline & & & Fig. S3-Ti & Titanium white & & & & \\
\hline & & & Fig. S3-Ba & Barium sulfate & & & & \\
\hline & & & Fig. S3-Co & \multirow{2}{*}{$\begin{array}{l}\text { Cobalt blue / cobalt } \\
\text { green } \\
\text { Zinc white / cobalt green }\end{array}$} & & & & \\
\hline & & & 119. & & & & & \\
\hline
\end{tabular}

Table 2. Overview of the materials identified or tentatively identified through non-invasive analysis of the six paintings investigated. Continued 


\begin{tabular}{|c|c|c|c|c|c|c|c|c|}
\hline \multirow[t]{2}{*}{ Painting } & \multirow[t]{2}{*}{ Color } & \multicolumn{3}{|l|}{ MA-XRF } & \multicolumn{4}{|c|}{ Reflectance spectroscopy } \\
\hline & & Element & $\begin{array}{l}\text { Figure/ } \\
\text { Distribution } \\
\text { map }\end{array}$ & $\begin{array}{l}\text { Tentative pigment } \\
\text { identification }\end{array}$ & $\begin{array}{l}\text { Distribution } \\
\text { map / } \\
\text { Spectrum }\end{array}$ & $\begin{array}{l}\text { Maximum } \\
\text { absorption } \\
(\mathrm{nm})\end{array}$ & $\begin{array}{l}\text { Inflection } \\
\text { point } \\
(\mathrm{nm})\end{array}$ & $\begin{array}{l}\text { Tentative } \\
\text { pigment } \\
\text { identification }\end{array}$ \\
\hline \multirow[t]{21}{*}{$\begin{array}{l}2004- \\
005\end{array}$} & Green & $\mathrm{Cr}$ & Fig. S5-Cr & $\begin{array}{l}\text { Viridian, } \\
\text { chrome(III)oxide green } \\
\text { and/or chrome yellow + } \\
\text { blue }\end{array}$ & $\begin{array}{l}\text { Fig. S6- } \\
\text { EM7 }\end{array}$ & $\begin{array}{l}450,600 \\
710\end{array}$ & 750 & $\begin{array}{l}\text { Viridian and/or } \\
\text { chrome(III)oxide } \\
\text { green }\end{array}$ \\
\hline & \multirow[t]{2}{*}{ Red } & \multirow[t]{2}{*}{$\mathrm{Hg}$} & \multirow[t]{2}{*}{ Fig. S5-Hg } & \multirow[t]{2}{*}{ Vermilion } & $\begin{array}{l}\text { Fig. S6- } \\
\text { EM5 }\end{array}$ & $\begin{array}{l}540,660 \\
700\end{array}$ & 580 & \multirow{2}{*}{$\begin{array}{l}\text { Mixture } \\
\text { containing } \\
\text { vermilion }\end{array}$} \\
\hline & & & & & $\begin{array}{l}\text { Fig. S6- } \\
\text { EM6 }\end{array}$ & & & \\
\hline & Yellow & $\mathrm{N} / \mathrm{A}$ & $\mathrm{N} / \mathrm{A}$ & Possibly organic & $\begin{array}{l}\text { Fig. S6- } \\
\text { EM1 }\end{array}$ & $\mathrm{N} / \mathrm{A}$ & $\mathrm{N} / \mathrm{A}$ & $\begin{array}{l}\text { Unknown } \\
\text { (possibly } \\
\text { organic) yellow }\end{array}$ \\
\hline & \multirow[t]{3}{*}{ Brown } & \multirow{3}{*}{$\begin{array}{l}\mathrm{Fe}, \mathrm{Mn} \text {, } \\
\mathrm{Hg}\end{array}$} & Fig. S5-Fe & Umber & \multirow{3}{*}{$\begin{array}{l}\text { Fig. S6- } \\
\text { EM2 }\end{array}$} & \multirow[t]{3}{*}{$\mathrm{N} / \mathrm{A}$} & \multirow[t]{3}{*}{$\mathrm{N} / \mathrm{A}$} & \multirow[t]{3}{*}{ Unknown dark } \\
\hline & & & Fig. S5-Mn & & & & & \\
\hline & & & Fig. S5-Hg & Vermilion & & & & \\
\hline & \multirow[t]{4}{*}{ Black } & \multirow[t]{4}{*}{$\begin{array}{l}\mathrm{Cr}, \mathrm{Ca} \\
\mathrm{Mn}, \mathrm{Hg}\end{array}$} & Fig. S5-Cr & $\begin{array}{l}\text { Viridian, } \\
\text { chrome(III)oxide green } \\
\text { and/or chrome yellow + } \\
\text { blue }\end{array}$ & \multirow[t]{4}{*}{$\mathrm{N} / \mathrm{A}$} & \multirow[t]{4}{*}{$\mathrm{N} / \mathrm{A}$} & \multirow[t]{4}{*}{$\mathrm{N} / \mathrm{A}$} & \multirow[t]{4}{*}{$\mathrm{N} / \mathrm{A}$} \\
\hline & & & Fig. S5-Ca & Bone black & & & & \\
\hline & & & Fig. S5-Mn & Umber & & & & \\
\hline & & & Fig. S5-Hg & Vermilion & & & & \\
\hline & \multirow[t]{3}{*}{ Ground } & $\mathrm{Pb}$ & Fig. S5-Pb & Lead white & \multirow[t]{3}{*}{$\mathrm{N} / \mathrm{A}$} & \multirow[t]{3}{*}{$\mathrm{N} / \mathrm{A}$} & \multirow[t]{3}{*}{$\mathrm{N} / \mathrm{A}$} & \multirow[t]{3}{*}{$\mathrm{N} / \mathrm{A}$} \\
\hline & & $\mathrm{Ca}$ & & Calcium carbonate & & & & \\
\hline & & $\mathrm{Ba}$ & & Barium sulfate & & & & \\
\hline & \multirow[t]{7}{*}{ Retouching } & \multirow{7}{*}{$\begin{array}{l}\mathrm{Ca}, \mathrm{Fe}, \mathrm{K} \\
\mathrm{Ti}, \mathrm{Ba} \\
\mathrm{Co}, \mathrm{Zn}\end{array}$} & Fig. S5-Mn & Umber (?) & \multirow{3}{*}{$\begin{array}{l}\text { Fig. S6- } \\
\text { EM3 }\end{array}$} & \multirow{3}{*}{$\begin{array}{l}630,705 \\
795\end{array}$} & & \multirow{3}{*}{$\begin{array}{l}\text { Possibly } \\
\text { organic green }\end{array}$} \\
\hline & & & Fig. S5-Ca & Calcium carbonate & & & & \\
\hline & & & Fig. S5-K & Unknown & & & & \\
\hline & & & Fig. S5-Ti & Titanium white & \multirow{4}{*}{$\begin{array}{l}\text { Fig. S6- } \\
\text { EM4 }\end{array}$} & 480,630 & & Ochre \\
\hline & & & Fig. S5-Ba & Barium sulfate & & & & \\
\hline & & & Fig. S5-Co & $\begin{array}{l}\text { Cobalt blue / cobalt } \\
\text { green }\end{array}$ & & & & \\
\hline & & & Fig. ১১-Ln & $\begin{array}{l}\text { Zinc white / cobalt } \\
\text { green }\end{array}$ & & & & \\
\hline
\end{tabular}

Table 2. Overview of the materials identified or tentatively identified through non-invasive analysis of the six paintings investigated. Continued 


\begin{tabular}{|c|c|c|c|c|c|c|c|c|}
\hline \multirow[t]{2}{*}{ Painting } & \multirow[t]{2}{*}{ Color } & \multicolumn{3}{|l|}{ MA-XRF } & \multicolumn{4}{|c|}{ Reflectance spectroscopy } \\
\hline & & Element & $\begin{array}{l}\text { Figure/ } \\
\text { Distribution } \\
\text { map }\end{array}$ & $\begin{array}{l}\text { Tentative pigment } \\
\text { identification }\end{array}$ & $\begin{array}{l}\text { Distribution } \\
\text { map / } \\
\text { Spectrum }\end{array}$ & $\begin{array}{l}\text { Maximum } \\
\text { absorption } \\
(\mathrm{nm})\end{array}$ & $\begin{array}{l}\text { Inflection } \\
\text { point } \\
(\mathrm{nm})\end{array}$ & $\begin{array}{l}\text { Tentative } \\
\text { pigment } \\
\text { identification }\end{array}$ \\
\hline \multirow[t]{14}{*}{$\begin{array}{l}2004- \\
007\end{array}$} & \multirow[t]{2}{*}{ Green } & \multirow[t]{2}{*}{$\begin{array}{l}\mathrm{Cu}, \mathrm{As}, \\
\mathrm{Cr}\end{array}$} & $\begin{array}{l}\text { Fig. S7-Cu } \\
\text { Fig. S7-As }\end{array}$ & $\begin{array}{l}\text { Emerald green and/or } \\
\text { Scheele's green }\end{array}$ & $\begin{array}{l}\text { Fig. S8- } \\
\text { EM2 }\end{array}$ & \multicolumn{2}{|l|}{430,680} & $\begin{array}{l}\text { Emerald } \\
\text { green }\end{array}$ \\
\hline & & & Fig. S7-Cr & $\begin{array}{l}\text { Viridian, chrome(III)oxide } \\
\text { green and/or chrome yellow } \\
+ \text { blue }\end{array}$ & $\begin{array}{l}\text { Fig. S8- } \\
\text { EM7 }\end{array}$ & 680 & 480 & $\begin{array}{l}\text { Prussian } \\
\text { blue + yellow }\end{array}$ \\
\hline & Blue & $\mathrm{N} / \mathrm{A}$ & N/A & N/A & $\begin{array}{l}\text { Fig. S8- } \\
\text { EM1 }\end{array}$ & 680 & & $\begin{array}{l}\text { Prussian } \\
\text { blue }\end{array}$ \\
\hline & Red & $\mathrm{Hg}$ & Fig. S7-Hg & Vermilion & $\begin{array}{l}\text { Fig. S8- } \\
\text { EM8 }\end{array}$ & N/A & 590 & Vermilion \\
\hline & Yellow & $\mathrm{Cr}$ & Fig. S7-Cr & Chrome yellow & N/A & N/A & $\mathrm{N} / \mathrm{A}$ & N/A \\
\hline & \multirow[t]{2}{*}{ Brown } & \multirow[t]{2}{*}{$\mathrm{Fe}, \mathrm{Mn}$} & & \multirow[t]{2}{*}{ Umber } & \multirow{2}{*}{$\begin{array}{l}\text { Fig. S8- } \\
\text { EM3 } \\
\text { Fig. S8- } \\
\text { EM4 }\end{array}$} & \multirow[t]{2}{*}{690} & \multirow[t]{2}{*}{575} & \multirow[t]{2}{*}{ Unknown } \\
\hline & & & rig. S /-IVIn & & & & & \\
\hline & \multirow[t]{2}{*}{ Black } & \multirow[t]{2}{*}{$\mathrm{Ca}, \mathrm{Co}$} & Fig. S7-Ca & Bone black & \multirow{2}{*}{$\begin{array}{l}\text { Fig. S8- } \\
\text { EM5 }\end{array}$} & \multirow[t]{2}{*}{ N/A } & \multirow[t]{2}{*}{$\mathrm{N} / \mathrm{A}$} & \multirow[t]{2}{*}{ Unknown } \\
\hline & & & Fig. S7-Co & Cobalt blue & & & & \\
\hline & \multirow[t]{4}{*}{ Ground } & \multirow{4}{*}{$\begin{array}{l}\mathrm{Pb}, \mathrm{Ba} \\
\mathrm{Zn}, \mathrm{Ca} \\
\mathrm{S}\end{array}$} & Fig. S7-Pb & Lead white & \multirow[t]{4}{*}{$\mathrm{N} / \mathrm{A}$} & \multirow[t]{4}{*}{$\mathrm{N} / \mathrm{A}$} & \multirow[t]{4}{*}{ N/A } & \multirow[t]{4}{*}{$\mathrm{N} / \mathrm{A}$} \\
\hline & & & Fig. S7-Ba & Barium sulfate & & & & \\
\hline & & & Fig. S7-Zn & Zinc oxide & & & & \\
\hline & & & Fig. S7-Ca & $\begin{array}{l}\text { Calcium carbonate and/or } \\
\text { sulfate }\end{array}$ & & & & \\
\hline & $\begin{array}{l}\text { Possible } \\
\text { retouching }\end{array}$ & $\mathrm{Zn}$ & Fig. S7-Zn & Zinc white & $\begin{array}{l}\text { Fig. S8- } \\
\text { EM6 }\end{array}$ & $\begin{array}{l}630,710 \\
795\end{array}$ & & $\begin{array}{l}\text { Possibly } \\
\text { modern } \\
\text { pigment }\end{array}$ \\
\hline
\end{tabular}

Table 2. Overview of the materials identified or tentatively identified through non-invasive analysis of the six paintings investigated. Continued 


\begin{tabular}{|c|c|c|c|c|c|c|c|c|}
\hline \multirow[t]{2}{*}{ Painting } & \multirow[t]{2}{*}{ Color } & \multicolumn{3}{|l|}{ MA-XRF } & \multicolumn{4}{|c|}{ Reflectance spectroscopy } \\
\hline & & Element & $\begin{array}{l}\text { Figure/ } \\
\text { Distribution } \\
\text { map }\end{array}$ & $\begin{array}{l}\text { Tentative pigment } \\
\text { identification }\end{array}$ & $\begin{array}{l}\text { Distribution } \\
\text { map / } \\
\text { Spectrum }\end{array}$ & $\begin{array}{l}\text { Maximum } \\
\text { absorption } \\
(\mathrm{nm})\end{array}$ & $\begin{array}{l}\text { Inflection } \\
\text { point } \\
(\mathrm{nm})\end{array}$ & $\begin{array}{l}\text { Tentative } \\
\text { pigment } \\
\text { identification }\end{array}$ \\
\hline \multirow{13}{*}{$\begin{array}{l}2003- \\
05-02\end{array}$} & \multirow[t]{2}{*}{ Green } & \multirow[t]{2}{*}{$\mathrm{Cr}, \mathrm{Cu}$} & Fig. S9-Cr & $\begin{array}{l}\text { Viridian, chrome(III)oxide green } \\
\text { and/or chrome yellow + blue }\end{array}$ & \multirow[t]{2}{*}{$\begin{array}{l}\text { Fig.S10- } \\
\text { EM4 }\end{array}$} & \multirow[t]{2}{*}{700} & \multirow[t]{2}{*}{505} & \multirow[t]{2}{*}{$\begin{array}{l}\text { Prussian } \\
\text { blue + yellow }\end{array}$} \\
\hline & & & Fig. S9-Cu & $\begin{array}{l}\text { Cu-based green such as } \\
\text { verdigris, copper resinate or } \\
\text { malachite }\end{array}$ & & & & \\
\hline & \multirow[t]{2}{*}{ Blue } & \multirow[t]{2}{*}{ N/A } & \multirow[t]{2}{*}{ N/A } & \multirow[t]{2}{*}{ N/A } & $\begin{array}{l}\text { Fig.S10- } \\
\text { EM1 }\end{array}$ & 700 & N/A & $\begin{array}{l}\text { Prussian } \\
\text { blue }\end{array}$ \\
\hline & & & & & $\begin{array}{l}\text { Fig.S10- } \\
\text { EM3 }\end{array}$ & 420,600 & 720 & $\begin{array}{l}\text { Ultramarine } \\
\text { blue }\end{array}$ \\
\hline & \multirow[t]{2}{*}{ Red } & \multirow[t]{2}{*}{$\mathrm{Fe}, \mathrm{Hg}$} & Fig. S9-Fe & $\begin{array}{l}\text { Iron oxide/ } \\
\text { oxy-hydroxide }\end{array}$ & $\begin{array}{l}\text { Fig.S10- } \\
\text { EM2 }\end{array}$ & $\begin{array}{l}480,650 \\
865\end{array}$ & 580 & Red ochre \\
\hline & & & Fig. S9-Hg & Vermilion & $\begin{array}{l}\text { Fig.S10- } \\
\text { EM5 }\end{array}$ & $\begin{array}{l}485,650 \\
900\end{array}$ & 585 & $\begin{array}{l}\text { Vermilion }(+ \\
\text { iron oxide) }\end{array}$ \\
\hline & \multirow[t]{2}{*}{ Yellow } & \multirow[t]{2}{*}{$\mathrm{Fe}, \mathrm{Cr}$} & Fig. S9-Fe & $\begin{array}{l}\text { Iron oxide/ } \\
\text { oxy-hydroxide }\end{array}$ & $\begin{array}{l}\text { Fig.S10- } \\
\text { EM6 }\end{array}$ & $\begin{array}{l}555,620 \\
850\end{array}$ & $\mathrm{~N} / \mathrm{A}$ & $\begin{array}{l}\text { Yellow ochre } \\
+ \text { other } \\
\text { yellow }\end{array}$ \\
\hline & & & Fig. S9-Cr & Chrome yellow & $\begin{array}{l}\text { Fig.S10- } \\
\text { EM8 }\end{array}$ & 480,620 & 520 & $\begin{array}{l}\text { Possibly } \\
\text { chrome } \\
\text { yellow }\end{array}$ \\
\hline & \multirow[t]{2}{*}{ Brown } & \multirow[t]{2}{*}{$\mathrm{Fe}, \mathrm{Mn}$} & Fig. S9-Fe & \multirow[t]{2}{*}{ Umber } & & & & \\
\hline & & & Fig. S9-Mn & & & & & \\
\hline & Black & $\mathrm{Ca}$ & Fig. S9-Ca & Bone black & $\begin{array}{l}\text { Fig.S10- } \\
\text { EM9 }\end{array}$ & 645 & N/A & Bone black \\
\hline & \multirow[t]{2}{*}{ Ground } & $\mathrm{Pb}$ & Fig. S9-Pb & Lead white & \multirow[t]{2}{*}{ N/A } & \multirow[t]{2}{*}{ N/A } & \multirow[t]{2}{*}{$\mathrm{N} / \mathrm{A}$} & \\
\hline & & $\mathrm{Zn}$ & & Zinc white & & & & \\
\hline
\end{tabular}

Table 3. Overview of the materials identified through minimally invasive analyses of the six investigated paintings. 


\begin{tabular}{|c|c|c|c|c|c|}
\hline Painting & Sample & \multicolumn{2}{|c|}{$\begin{array}{l}\text { Analytical technique and associated element } \\
\text { (SEM-EDX) and Raman shifts }\left(\mathrm{cm}^{-1}\right) \text {. }\end{array}$} & Figure & Identified materials in ground and colored layers \\
\hline \multirow[t]{3}{*}{ Trapiche } & \multirow[t]{3}{*}{ CS1 } & SEM-EDX & $\mathrm{Co}, \mathrm{Al}, \mathrm{Zn}, \mathrm{Pb}$ & Fig. 5 & Ground: N/A \\
\hline & & \multirow[t]{2}{*}{ Raman } & $\begin{array}{l}328,434,545,567,1050,1090- \\
1142\end{array}$ & $\begin{array}{l}\text { Fig. 6- } \\
\text { a }\end{array}$ & \multirow[t]{2}{*}{$\frac{\text { Colored layer: cobalt blue, cobalt green and lead }}{\text { white }}$} \\
\hline & & & 202,513 & $\begin{array}{l}\text { Fig. 6- } \\
\text { b }\end{array}$ & \\
\hline \multirow{4}{*}{$\begin{array}{l}\text { CAM- } \\
1329\end{array}$} & \multirow[t]{3}{*}{ SCR1 } & SEM-EDX & $\mathrm{Pb}, \mathrm{Ba}$ & $\mathrm{N} / \mathrm{A}$ & \multirow{4}{*}{$\begin{array}{l}\text { Ground: N/A } \\
\text { Colored layer: Lead white, vermilion, and Ba- } \\
\text { containing yellow, likely organic, identified as } \\
\text { PY1 (Hansa Yellow G - C.I. 11680) }\end{array}$} \\
\hline & & \multirow[t]{2}{*}{ Raman } & 1050 & $\begin{array}{l}\text { Fig. } \\
\text { S14-j }\end{array}$ & \\
\hline & & & $250,281,340$ & $\begin{array}{l}\text { Fig. } \\
\text { S14-k }\end{array}$ & \\
\hline & SCR2 & $\begin{array}{l}\text { HPLC-DAD- } \\
\mathrm{MS} / \mathrm{MS}\end{array}$ & & Fig. 8 & \\
\hline \multirow{7}{*}{$\begin{array}{l}\text { CAM- } \\
1328\end{array}$} & \multirow[t]{6}{*}{$\mathrm{cs} 2$} & SEM-EDX & $\mathrm{Pb}, \mathrm{Cr}, \mathrm{Cu}, \mathrm{As}, \mathrm{Ca}, \mathrm{Al}, \mathrm{Mg}$ & Fig. 7 & \multirow{6}{*}{$\begin{array}{l}\text { Ground: lead white } \\
\text { Colored layer: ultramarine blue, viridian, Emerald } \\
\text { green, and vermilion }\end{array}$} \\
\hline & & \multirow[t]{5}{*}{ Raman } & 1050 & $\begin{array}{l}\text { Fig. 6- } \\
\text { c }\end{array}$ & \\
\hline & & & $260,345,432,487,541,583,837$ & $\begin{array}{l}\text { Fig. 6- } \\
\text { d }\end{array}$ & \\
\hline & & & $\begin{array}{l}106,153,175,217,242,292,325 \\
371,429,541\end{array}$ & $\begin{array}{l}\text { Fig. 6- } \\
\text { e }\end{array}$ & \\
\hline & & & $256,546,581,804,1092$ & $\begin{array}{l}\text { Fig. 6- } \\
f\end{array}$ & \\
\hline & & & $252,284,342$ & $\begin{array}{l}\text { Fig. 6- } \\
\mathrm{g}\end{array}$ & \\
\hline & SCR3 & $\begin{array}{l}\text { HPLC-DAD- } \\
\text { MS/MS }\end{array}$ & & Fig. 9 & PY3 (Hansa Yellow 10G - C.I. 11710) \\
\hline \multirow[t]{3}{*}{$\begin{array}{l}2004- \\
005\end{array}$} & \multirow[t]{3}{*}{ CS3 } & SEM-EDX & $\mathrm{Ba}, \mathrm{Ca}, \mathrm{Pb}, \mathrm{Fe}$ & $\begin{array}{l}\text { Fig. } \\
\text { S11 }\end{array}$ & \multirow{3}{*}{$\begin{array}{l}\text { Ground: likely lead white, likely calcium carbonate, } \\
\text { and barium sulfate } \\
\text { Pigmented layer 1 }(\underline{\text { top}}) \text { ): Ba-rich light yellow } \\
\text { Pigmented layer } 1 \text { (ㅁottom): Mars red }\end{array}$} \\
\hline & & \multirow[t]{2}{*}{ Raman } & $224,290,410,496,610$ & $\begin{array}{l}\text { Fig. } \\
\text { S14-a }\end{array}$ & \\
\hline & & & $453,461,986$ & $\begin{array}{l}\text { Fig. } \\
\text { S14-b }\end{array}$ & \\
\hline \multirow[t]{7}{*}{$\begin{array}{l}2004- \\
007\end{array}$} & \multirow[t]{7}{*}{ cS4 } & \multirow[t]{2}{*}{ SEM-EDX, } & Ground: $\mathrm{Zn}, \mathrm{Ba}, \mathrm{S}, \mathrm{Ca}, \mathrm{Pb}, \mathrm{Si}$ & $\begin{array}{l}\text { Fig. } \\
\text { S12 }\end{array}$ & \multirow{7}{*}{$\begin{array}{l}\text { Ground: lead white, calcium carbonate, calcium } \\
\text { sulfate, quartz, and lithopone } \\
\text { Colored layer: lead white, Prussian blue, and } \\
\text { vermilion }\end{array}$} \\
\hline & & & 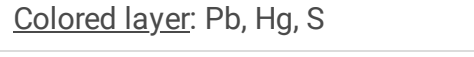 & & \\
\hline & & \multirow[t]{5}{*}{ Raman } & 453,986 & $\begin{array}{l}\text { Fig. } \\
\text { S14-c }\end{array}$ & \\
\hline & & & $250,281,340$ & $\begin{array}{l}\text { Fig. } \\
\text { S14-d }\end{array}$ & \\
\hline & & & $154,281,1085$ & $\begin{array}{l}\text { Fig. } \\
\text { S14-e }\end{array}$ & \\
\hline & & & $127,202,463$ & $\begin{array}{l}\text { Fig. } \\
\text { S14-f }\end{array}$ & \\
\hline & & & 1006 & $\begin{array}{l}\text { Fig. } \\
\text { S14-g }\end{array}$ & \\
\hline \multirow[t]{3}{*}{$\begin{array}{l}2003- \\
05-02\end{array}$} & \multirow[t]{3}{*}{ CS5 } & SEM-EDX & $\mathrm{Pb}, \mathrm{Zn}, \mathrm{Fe}, \mathrm{Al}$ & $\begin{array}{l}\text { Fig. } \\
\text { S13 }\end{array}$ & \multirow{3}{*}{$\begin{array}{l}\text { Ground: lead white and zinc white, with inclusion o } \\
\text { yellow ochre/goethite } \\
\text { Colored layer: N/A }\end{array}$} \\
\hline & & \multirow[t]{2}{*}{ Raman } & 1050 & $\begin{array}{l}\text { Fig. } \\
\text { S14-h }\end{array}$ & \\
\hline & & & $250,300,387$ & $\begin{array}{l}\text { Fig. } \\
\text { S14-i }\end{array}$ & \\
\hline
\end{tabular}


Micro-invasive investigation: optical microscopy, SEM-EDX, and Raman spectroscopy.

Optical microscopy, SEM-EDX mappings and Raman spectroscopy undertaken on embedded samples allowed for a better understanding of the paint stratigraphy as well as the distribution of the pigments and their identification. SEM-EDX and Raman allowed for the confirmation of the pigments tentatively identified using non-invasive imaging techniques and reported in Table 2 along with gaining insights into the nature of the ground used by the artists.

The sample taken from the light blue sky of Trapiche Meladero presents Co/Al-rich blue and Co/Zn-rich green coarse pigment particles dispersed in a Pb-rich white matrix (Fig. 5). Using Raman spectroscopy, the Pb-rich white matrix was identified as lead white through its bands at $1050 \mathrm{~cm}^{-1}$ (Fig. 6-a), whereas the Co/Zn-rich green and the Co/Al-rich blue particles were identified as cobalt green (Fig. 6-a) and cobalt blue (Fig. 6-b), respectively (Table 3). The positive identification of $\mathrm{Co} / \mathrm{Zn}$-rich cobalt green in mixture with a blue pigment in Trapiche Meladero could support the use of the same cobalt green pigment in mixture with Cr-based and Cu-based green hypothesized in CAM1329 (see section “Non-invasive imaging techniques: MA-XRF and RIS").

While inferred with MA-XRF and SEM-EDX (Fig. S3 and Fig. 7), a lead white ground was identified in the cross section taken from CAM-1328 based on its $1050 \mathrm{~cm}^{-1}$ characteristic Raman band (Fig. 6-C). Furthermore, Cr-based and Cu/As-based greens, also inferred using non-invasive imaging spectroscopies, were also observed with SEM-EDX in the green particles of CS2 (Fig. 7). These pigments were then respectively identified as viridian (Fig. 6-d) and Emerald green (Fig. 6-e) based on their characteristic Raman shift reported in Table 3. Though viridian refers to the hydrated chrome oxide form $\left(\mathrm{Cr}_{2} \mathrm{O}_{3} \cdot 2 \mathrm{H}_{2} \mathrm{O}\right)$, the spectrum reported in Fig. 6-d presents bands characteristic for both the hydrated (viridian; 583,487 and $260 \mathrm{~cm}^{-1}$ ) and the anhydrous oxide (chrome(III)oxide, 541 and $345 \mathrm{~cm}^{-1}$ ). Such features for viridian have been reported before [39].

Both techniques also allowed for the identification of ultramarine blue, based on the presence of Al and Si in the EDX spectrum (Fig. 7) and its 256, 546 and $804 \mathrm{~cm}^{-1}$ vibration bands in the Raman spectrum (Fig. 6-f). Vermilion was confirmed in the red particles (not observed with SEMEDX but identified by Raman through the 252, 284 and $342 \mathrm{~cm}^{-1}$ characteristic bands, Fig. 6-g).

Raman shifts, elements detected using SEM-EDX, and the associated pigment identifications for all five cross sections investigated are summarized in Table 3.

The yellow area of CS2 (Fig. 7) did not yield any elemental response expected for inorganic yellow pigments such as Cr or Zn. A similar observation was made for CS3 (Fig. S11) for which the lower darker reddish-yellow layer contained iron and was identified as Mars red by Raman (Fig. S14-a) whereas the upper light-yellow layer only contained Ba and could not be identified with spectroscopic techniques (Fig. S11). The yellow colors found in Oller's paintings, which did not present any elemental response in MA-XRF (knife handle, avocado flesh, bowl content, Fig. 2, and Fig. S3) were further investigated using the scrapings taken in the above-mentioned areas (Fig. 1). The SEM-EDX from the knife handle showed a mixture of $\mathrm{Pb}$ and $\mathrm{Ba}$ (data not shown) but confirmed the lack of elements usually associated with inorganic yellow, further suggesting the use of an organic yellow pigment or lake. However, Raman spectroscopy, which is well suited for the investigation of synthetic organic pigments [40-42], did not produce any spectra which would enable the pigments/colorants to be identified. This further emphasized the need for additional analysis using HPLC-DAD-MS/MS.

Micro-invasive chromatographic analysis: HPLC-DAD-MS/MS

The two yellow samples (SCR2 and SCR3) analyzed by HPLC-DAD-MS/MS revealed the use of two synthetic organic pigments in paintings CAM1329 and CAM1328.

For sample SCR2 (CAM1329) the DAD chromatogram acquired at $350 \mathrm{~nm}$ showed a single peak at ca. $9.8 \mathrm{~min}$ (Fig. 8-a), corresponding to a compound with a characteristic UV-Vis absorption spectrum showing two distinct absorption maxima at ca. 340 and $410 \mathrm{~nm}$ (Fig. 8-a - insert). The mass spectrometric data revealed that the compound produced a positive ion $[\mathrm{M}+\mathrm{H}]^{+}$at $m / z 341.124$ (Fig. 8-b), but no clear mass was detected in negative ionization mode. The tandem mass spectrum was recorded in positive ionization mode (Fig. 8-c). The results, specifically the retention time, UV-Vis absorption spectrum, accurate mass and tandem mass spectrum, were in perfect agreement with the analyses carried out on a reference sample of PY1 (Hansa Yellow G - C.I. 11680). The molecule ionizes in negative mode as well, producing a [M-H] ion at $m / z$ 339.110 , although the intensity of the negative ion is lower than the intensity of the corresponding positive ion. This explains the result obtained for sample SCR2, as the concentration of the molecule extracted from the tiny sample was not sufficient to produce a detectable negative ion. The results obtained for the reference sample of PY1, including the tandem mass spectrum obtained in negative ionization mode, are reported 
in Supplementary Information (Fig. S15) together with a brief discussion of the mass fragmentations observed in both positive and negative ionization modes.

For sample SCR3 (CAM1328) the DAD chromatogram acquired at $350 \mathrm{~nm}$ showed a single peak at ca. 10.4 min (Fig. 9-a), corresponding to a compound with a very similar UV-Vis absorption spectrum to what observed for sample SCR2, also showing two distinct absorption maxima at ca. 340 and $410 \mathrm{~nm}$ (Fig. 9-a - insert). In addition to the different retention time, the compound produced a positive ion [M+H] ${ }^{+}$at $m / z 395.031$ (Fig. 9-b). Also in this case, no clear mass was detected in negative ionization mode. The tandem mass spectrum was therefore recorded in positive ionization mode (Fig. 9-C). The results, specifically the retention time, UV-Vis absorption spectrum, accurate mass and tandem mass spectrum, were in perfect agreement with the analyses carried out on a reference sample of PY3 (Hansa Yellow 10G - C.I. 11710). Similarly to PY1, the molecule ionizes in negative mode as well, producing a [M-H] ion at $\mathrm{m} / \mathrm{z} 393.016$, with a lower intensity compared to the positive ion, again explaining the lack of detection of the deprotonated molecule in sample SCR3, due to a very low concentration. The results obtained for the reference sample of PY3, including the tandem mass spectrum obtained in negative ionization mode, are reported in Supplementary Information (Fig. S16) together with a brief discussion of the mass fragmentations observed in both positive and negative ionization modes.

\section{Discussion}

Most of the pigments used by Oller, Cuchí y Arnau, and Frade and identified during this study are similar to the ones used by French and American impressionist and post-impressionist artists of the time $[6,7,9,10,36,43-48]$. As described in the results section and summarized in Tables 2 and 3, these pigments include hydrated chromium oxide - known as viridian, and Emerald green for the greens; Prussian blue, cobalt blue and ultramarine blue for the blues; vermilion and red ochre for the reds and carnation; yellow ochres and chrome yellow for the yellows; umber or sienna for the browns; cobalt blue and possibly bone/ivory black as constituents of the darker and black passages. Lead and zinc were identified in white areas and indicate the use of lead white and zinc white, respectively. Mixtures of aforementioned pigments have been identified and are reported in Table 2. Green pigments such as viridian and Emerald green are often reported in most technical studies of impressionist and post-impressionist artists such as Monet, Renoir, Pissarro, and Munch. This is to be expected as these pigments are often listed in most major paint manufacturing companies such as Winsor \& Newton, Bourgeois Ainé, and Lefranc [49]. Consequently, due to their ties with European artists, such pigments could be expected as part of the Puerto Rican artists' palettes. However, despite the large correspondence between pigments used by these three Puerto Rican artists and their European and American contemporaries, both non-invasive and minimally invasive analyses highlighted the use of cobalt green and synthetic organic yellows, pigments usually not reported in technical studies of paintings from this time period.

The use of cobalt green, a cobalt oxide-doped zinc oxide green pigment, appears unexpected. Indeed, while invented in 1780 by Swedish Chemist Sven Rinmann, the pigment is rarely reported in technical studies of late $19^{\text {th }}$ century artists. Despite its permanence, the pigment was unpopular upon its discovery due to its high cost and poor tinting strength [50]. It was described as "chemically good and artistically bad", which may explain its apparent restricted use in impressionist and post-impressionist paintings. Despite the overall lack of identification in technical studies, cobalt green was recently found in paintings by French artists James Tissot [51]. Furthermore, the pigment was also advertised as part of the pigment catalogue of major paint manufacturing companies such as Winsor \& Newton, Bourgeois Ainé, and Lefranc, vouching for its availability to artists [49]. Therefore, its use in late $19^{\text {th }}$ century paintings may be more widespread than anticipated and its lack of identification may be attributed to the limitations of non-invasive techniques such as XRF or reflectance spectroscopy. Indeed, with the aforementioned techniques, the pigment may be mistaken for a mixture of zinc white and cobalt blue, two common pigments of this time period. More technical studies of impressionists and post-impressionist paintings with non-invasive and minimally invasive techniques would be require to confirm this hypothesis. This is however outside of the scope of this article.

The use of synthetic yellow pigments in CAM-1328 and CAM1329, hypothesized upon non-invasive analyses, and identified with HPLC-DADMS/MS as PY1 (Hansa Yellow G - C.I. 11680) and PY3 (Hansa Yellow 10G - C.I. 11710) was also unexpected. Indeed, chrome yellow, cadmium yellow, iron oxide, zinc yellow, strontium yellow, barium yellow and Naples yellow, all widely available from major paint manufacturing companies [49] were often identified in paintings from this time period [7,43,46,52]. In that regard, the use of chrome yellow in in 2004-007 and 2003-05-02 (Fig. S7, and Fig. S9) corresponds better to the European practices of this time period. Nonetheless, the use of synthetic organic pigments cannot be excluded in late- $19^{\text {th }} /$ early $20^{\text {th }}$ century paintings as this period saw the development of many organic pigments including some organic yellows such as tartrazine (PY100 - C.I. 19140:1), discovered in 1884 by H. Ziegleras [50,53] as well as several Hansa yellows in the early 1900s [42,53]. Both PY1 and PY3 are described as light-valued opaque to semitransparent intense yellows, therefore making them suitable alternative to bright chrome yellow [54]. PY3, identified in the avocado flesh of CAM-1328, is also described as bright greenish yellow [53], making it suitable to depict the greenish hue of the avocado flesh. While both PY1 and PY3 may not appear as immediate candidates to impressionist and post-impressionist paintings, they were respectively discovered in 1909-10 and 1910-11 [53,55] and started to be produced in 1910 [55], therefore during the lifetime of Francisco Oller (1833-1917). 
Despite the current lack of historical notes, it is known that Ricardo Alegría (1921-2011), a Puerto Rican historian, cultural anthropologist and archaeologist, and Osiris Delgado (1920-2017), a painter, writer, and art historian also from Puerto Rico, used biographical and historical data to date the paintings from circa 1890 (CAM-1329) and 1890-91 (CAM-1328), as indicated in the museum's records. This approach therefore dated the paintings 20 years prior to the discovery and development of PY1 and PY3. Nonetheless, the pigments were available prior to Oller's death in 1917, with the artist known for having been active until the end of his life creating paintings such as Guayabas being dated 1901-03; Higueras being dated ca. 1912; and Piñas dated 1912-14 [56]. Furthermore, close visual examinations do not present signs of losses and overpaints in the yellow areas analyzed, suggesting the yellows areas to be original. As a result, the presence of these pigments (PY1 and PY3) allows us to change the terminus post quem of CAM-1328 and CAM-1329 to 1910, further emphasizing Oller's activity until late in his career. This also illustrates the importance of scientific analyses along with the connoisseurship approach to better comprehend the history associated with artworks, especially when these are not explicitly dated, and help refine the information associated with the artwork, which is then made available to the public and scholars.

\section{Conclusion}

This technical study aimed at characterizing Puerto Rican artists' palette for the first time. Due to the relationship Puerto Rico has had with Europe until the late $19^{\text {th }}$ century and the transatlantic travels of the Puerto Rican artists, they represent key figures to understand the influence of European practices in the Caribbean.

Non-invasive techniques (namely MA-XRF and RIS), coupled with minimally invasive techniques such as SEM-EDX, Raman and HPLC-DADMS/MS allowed for the characterization of the artists' palettes. While most artists made use of pigments traditionally employed in the late $19^{\text {th }}$ century (lead white, zinc white, cobalt blue, Prussian blue, ultramarine blue, Emerald green, viridian, vermilion, red iron oxide, umber, yellow iron oxide, chrome yellow, bone black), this study highlighted the use of organic yellow pigments (PY1 and PY3) and cobalt green, a notoriously expensive and unpopular pigment due to its poor tinting strength. The use of organic yellows instead of usual inorganic yellow pigments is unexpected, as they are rarely found in technical studies of late $19^{\text {th }} /$ early $20^{\text {th }}$ century paintings and inorganic yellow pigments were widely available from paint manufacturers. The use of PY1 and PY3, with well documented discovery and production dates, also allowed to redefine the realization dates of the two paintings in which they were found from 1890-91 to post-1910, closer to the end of Francisco Oller's career. Finally, cobalt green, while never part of pigments identified in technical studies of impressionist and post-impressionist paintings, was identified in two successive NU-ACCESS projects, which may indicate that it was more widely used than one would think, but its identification went undetected due to the pigment sharing its elemental constitution with cobalt blue and zinc white, widely used at the time. These results clearly support the importance of integrated studies including non-invasive, imaging techniques along with minimally invasive molecular techniques, thus providing a full understanding of the nature and spatial distribution of inorganic and organic materials used in paintings.

\section{Declarations}

List of abbreviations: MA-XRF, RIS, BSE, SEM-EDX, HPLC-DAD-MS/MS

Availability of data and materials: The datasets used analyzed during the current study are available from the corresponding author on reasonable request.

Competing interests: The authors declare that they have no competing interests

Funding: Andrew W. Mellon Foundation

Authors Contributions: M.V. wrote the manuscript with contributions from all authors. M.V., A.O.M, D.T., M.W., and S.R. carried out the noninvasive analysis. M.V. performed the invasive microanalysis. D.T. undertook the chromatographic analysis. M.W. served as the principal investigator for the project and provided overall supervision.

\section{Acknowledgments:}

This collaborative initiative is part of NU-ACCESS's broad portfolio of activities, made possible by generous support of the Andrew W. Mellon Foundation as well as supplemental support provided by the Materials Research Center, the Office of the Vice President for Research, the McCormick School of Engineering and Applied Science and the Department of Materials Science and Engineering at Northwestern University. This work made use of the EPIC and SPID facilities of Northwestern University's NUANCE Center, which has received support from the SHyNE Resource (NSF ECCS-2025633), the IIN, and Northwestern's MRSEC program (NSF DMR-1720139).

\section{References}


1. Sullivan, E.J. From San Juan to Paris and back : Francisco Oller and Caribbean art in the era of impressionism; Yale University Press: New Haven, 2014.

2. Nuño, J.A.G. La pintura puertorriqueña durante il signlo XIX. Revista del Instituto de Cultura Puertorriqueña 1979, 84, 22-35.

3. Mergal, A.M. Frade puertorriqueño pintor. Palique 1954.

4. Cottington, D. Cubism in the shadow of war: the avant-garde and politics in Paris 1905-1914; Yale University Press: New Haven [Conn, 1998.

5. Antliff, M. Inventing Bergson : cultural politics and the Parisian avant-garde, Princeton University Press: Princeton, N.J, 1993.

6. Roy, A. The Palettes of Three Impressionist Paintings. National Gallery Technical Bulletin 1985, 9, 12-20.

7. Roy, A. Monet's Palette in the Twentieth Century: 'Water-Lilies' and 'Irises'. National Gallery Technical Bulletin 2007, $28,58-68$.

8. Pozzi, F.; van den Berg, K.J.; Fiedler, I.; Casadio, F. A systematic analysis of red lake pigments in French Impressionist and PostImpressionist paintings by surface-enhanced Raman spectroscopy (SERS). Journal of Raman Spectroscopy 2014, 45, 1119-1126, doi:https://doi.org/10.1002/jrs.4483.

9. Pollack, M. Odilon Redon, Paul Gauguin, and Primitivist Color. The Art Bulletin 2020, 102, 77-103, doi:10.1080/00043079.2020.1711488.

10. Thurrowgood, D.; Paterson, D.; de Jonge, M.D.; Kirkham, R.; Thurrowgood, S.; Howard, D.L. A Hidden Portrait by Edgar Degas. Sci Rep 2016, 6, 10, doi:10.1038/srep29594.

11. Hermens, E.; Wallert, A. James McNeill Whistler: fluidity, finish and experiment. In Proceedings of the Studying old master paintings : technology and practice : the National Gallery Technical Bulletin 30th anniversary conference, London, 2011; pp. $229-236$.

12. Mazzinghi, A.; Ruberto, C.; Castelli, L.; Ricciardi, P.; Czelusniak, C.; Giuntini, L.; Mandò, P.A.; Manetti, M.; Palla, L.; Taccetti, F. The importance of being little: MA-XRF on manuscripts on a Venetian island. X-Ray Spectrometry 2021, 50, 272-278, doi:https://doi.org/10.1002/xrs.3181.

13. Delaney, J.K.; Conover, D.M.; Dooley, K.A.; Glinsman, L.; Janssens, K.; Loew, M. Integrated X-ray fluorescence and diffuse visible-to-nearinfrared reflectance scanner for standoff elemental and molecular spectroscopic imaging of paints and works on paper. Heritage Science 2018, 6, 31, doi:10.1186/s40494-018-0197-y.

14. Ricciardi, P.; Legrand, S.; Bertolotti, G.; Janssens, K. Macro X-ray fluorescence (MA-XRF) scanning of illuminated manuscript fragments: potentialities and challenges. Microchemical Journal 2016, 124, 785-791, doi:10.1016/j.microc.2015.10.020.

15. Dal Fovo, A.; Mazzinghi, A.; Omarini, S.; Pampaloni, E.; Ruberto, C.; Striova, J.; Fontana, R. Non-invasive mapping methods for pigments analysis of Roman mural paintings. Journal of Cultural Heritage 2020, 43, 311-318, doi:https://doi.org/10.1016/j.culher.2019.12.002.

16. Delaney, J.K.; Dooley, K.A.; van Loon, A.; Vandivere, A. Mapping the pigment distribution of Vermeer's Girl with a Pearl Earring. Heritage Science 2020, 8, 4, doi:10.1186/s40494-019-0348-9.

17. Aceto, M.; Agostino, A.; Fenoglio, G.; Idone, A.; Gulmini, M.; Picollo, M.; Ricciardi, P.; Delaney, J.K. Characterisation of colourants on illuminated manuscripts by portable fibre optic UV-visible-NIR reflectance spectrophotometry. Analytical Methods 2014, 6, doi:10.1039/c3ay41904e.

18. Baddini, A.L.d.Q.; Santos, J.L.V.d.P.; Tavares, R.R.; Paula, L.S.d.; Filho, H.d.C.A.; Freitas, R.P. PLS-DA and data fusion of visible Reflectance, XRF and FTIR spectroscopy in the classification of mixed historical pigments. Spectrochimica Acta Part A: Molecular and Biomolecular Spectroscopy 2022, 265, 120384, doi:https://doi.org/10.1016/j.saa.2021.120384.

19. Delaney, J.K.; Thoury, M.; Zeibel, J.G.; Ricciardi, P.; Morales, K.M.; Dooley, K.A. Visible and infrared imaging spectroscopy of paintings and improved reflectography. Heritage Science 2016, 4, 6, doi:10.1186/s40494-016-0075-4.

20. Glinsman, L. The application of X-ray fluorescence spectrometry to the study of museum objects. University of Amsterdam, Amsterdam, 2004.

21. Pouyet, E.; Brummel, K.; Webster-Cook, S.; Delaney, J.; Dejoie, C.; Pastorelli, G.; Walton, M. New insights into Pablo Picasso's La Miséreuse accroupie (Barcelona, 1902) using X-ray fluorescence imaging and reflectance spectroscopies combined with micro-analyses of samples. SN Applied Sciences 2020, 2, 1408, doi:10.1007/s42452-020-3130-4.

22. Vermeulen, M.; Tamburini, D.; Müller, E.M.K.; Centeno, S.A.; Basso, E.; Leona, M. Integrating liquid chromatography mass spectrometry into an analytical protocol for the identification of organic colorants in Japanese woodblock prints. Sci Rep 2020, 10, 20921, doi:10.1038/s41598-020-77959-2.

23. Wainwright, L. Americocentrism and Art of the Caribbean: Contours of a Time-Space Logic. Journal of American Studies 2013, 47, 417438, doi:10.1017/S0021875813000145.

24. Casey, E. Visual Culture of the Atlantic World. Heilbrunn Timeline of Art History 2018.

25. Hew, D. Afro-Caribbean Art of Jamaica. Emancipation 2001.

26. Cullen, D.; Fuentes Rodríguez, E. Caribbean : art at the crossroads of the world; El Museo del Barrio: New York, 2012.

27. Kriz, K.D. Slavery, sugar, and the culture of refinement : picturing the British West Indies, 1700-1840; Yale University Press: New Haven, 2008.

Page 16/24 
28. Landau, E.G. Mexico and American modernism; Yale University Press: New Haven, 2013.

29. Flores, T. Mexico's revolutionary avant-gardes : from Estridentismo to ;30-30!; Yale University Press: New Haven, 2013.

30. Duany, J. Picturing Cuba: Art, Culture, and Identity on the Island and in the Diaspora; University Press of Florida: Gainesville, 2019.

31. Victorian Jamaica; Duke University Press: 2018.

32. Pouyet, E.; Barbi, N.; Chopp, H.; Healy, O.; Katsaggelos, A.; Moak, S.; Mott, R.; Vermeulen, M.; Walton, M. Development of a highly mobile and versatile large MA-XRF scanner for in situ analyses of painted work of arts. X-Ray Spectrometry 2020, $n / a$, doi:https://doi.org/10.1002/xrs.3173.

33. Solé, V.A.; Papillon, E.; Cotte, M.; Walter, P.; Susini, J. A multiplatform code for the analysis of energy-dispersive X-ray fluorescence spectra. Spectrochimica Acta Part B: Atomic Spectroscopy 2007, 62, 63-68, doi:https://doi.org/10.1016/j.sab.2006.12.002.

34. Schindelin, J.; Arganda-Carreras, I.; Frise, E.; Kaynig, V.; Longair, M.; Pietzsch, T.; Preibisch, S.; Rueden, C.; Saalfeld, S.; Schmid, B.; et al. Fiji: an open-source platform for biological-image analysis. Nature Methods 2012, 9, 676-682, doi:10.1038/nmeth.2019.

35. Preibisch, S.; Saalfeld, S.; Tomancak, P. Globally optimal stitching of tiled 3D microscopic image acquisitions. Bioinformatics 2009, 25, 1463-1465, doi:10.1093/bioinformatics/btp184.

36. Vermeulen, M.; Smith, K.; Eremin, K.; Rayner, G.; Walton, M. Application of Uniform Manifold Approximation and Projection (UMAP) in spectral imaging of artworks. Spectrochimica Acta Part A: Molecular and Biomolecular Spectroscopy 2021, 252, 119547, doi:https://doi.org/10.1016/j.saa.2021.119547.

37. Vermeulen, M.; Müller, E.M.K.; Leona, M. Non-Invasive Study of the Evolution of Pigments and Colourants Use in 19th Century Ukiyo-e. Arts of Asia 2020, 50.

38. IFAC/CNR. Fiber Optics Reflectance Spectra (FORS) of Pictorial Materials in the 270-1700 nm range. 2020.

39. Coccato, A.; Bersani, D.; Coudray, A.; Sanyova, J.; Moens, L.; Vandenabeele, P. Raman spectroscopy of green minerals and reaction products with an application in Cultural Heritage research. Journal of Raman Spectroscopy 2016, 47, 1429-1443, doi:https://doi.org/10.1002/jrs.4956.

40. Saverwyns, S. Russian avant-garde... or not? A micro-Raman spectroscopy study of six paintings attributed to Liubov Popova. Journal of Raman Spectroscopy 2010, 41, 1525-1532, doi:10.1002/jrs.2654.

41. Fremout, W.; Saverwyns, S. Identification of synthetic organic pigments: the role of a comprehensive digital Raman spectral library. Journal of Raman Spectroscopy 2012, 43, 1536-1544, doi:https://doi.org/10.1002/jrs.4054.

42. Scherrer, N.C.; Stefan, Z.; Francoise, D.; Annette, F.; Renate, K. Synthetic organic pigments of the 20th and 21 st century relevant to artist's paints: Raman spectra reference collection. Spectrochimica Acta Part A: Molecular and Biomolecular Spectroscopy 2009, 73, 505-524, doi:https://doi.org/10.1016/j.saa.2008.11.029.

43. Ganz, J.A.; Groom, G.; Ireson, N.; Jaros, D.; Muir, K.; Nichols, K.; Shaw, J. Monet Paintings and Drawings at the Art Institute of Chicago; The Art Institute of Chicago: Chicago, 2014.

44. Walter, P.; Sarrazin, P.; Gailhanou, M.; Hérouard, D.; Verney, A.; Blake, D. Full-field XRF instrument for cultural heritage: Application to the study of a Caillebotte painting. X-Ray Spectrometry 2019, 48, 274-281, doi:https://doi.org/10.1002/xrs.2841.

45. Schaefer, I.; Lewerentz, K.; Saint-George, C.v. Painting light: the hidden techniques of the Impressionists, Skira ; Distributed in North America by Rizzoli International Publications: Milano, Italy : New York, NY, 2008; p. 238 p.

46. Burnstock, A.; Gutiérrez, L. Technical Examination of Works by Camille and Lucien Pissarro from the Courtauld Gallery. 2013.

47. Centeno, S.A.; Hale, C.; Carò, F.; Cesaratto, A.; Shibayama, N.; Delaney, J.; Dooley, K.; van der Snickt, G.; Janssens, K.; Stein, S.A. Van Gogh's Irises and Roses: the contribution of chemical analyses and imaging to the assessment of color changes in the red lake pigments. Heritage Science 2017, 5, 18, doi:10.1186/s40494-017-0131-8.

48. Monico, L.; Janssens, K.; Miliani, C.; Brunetti, B.G.; Vagnini, M.; Vanmeert, F.; Falkenberg, G.; Abakumov, A.; Lu, Y.; Tian, H.; et al. Degradation process of lead chromate in paintings by Vincent van Gogh studied by means of spectromicroscopic methods. 3. Synthesis, characterization, and detection of different crystal forms of the chrome yellow pigment. Anal Chem 2013, 85, 851-859, doi:10.1021/ac302158b.

49. Lizun, D.; Kurkiewicz, T.; Szczupak, B. Exploring Liu Kang's Paris Practice (1929-1932): Insight into Painting Materials and Technique. Heritage 2021, 4, 828-863.

50. CAMEO. The Conservation and Art Materials Encyclopedia Online (CAMEO). Available online: http://cameo.mfa.org/wiki/Tartrazine (accessed on 1/10/2022).

51. Kleiner, S.; Pouyet, E.; Oakley, L. Tissot's Painting Technique. In James Tissot, Buron, M.E., Ed.; PRESTEL: San Francisco, $2019 ;$ pp. $238-245$.

52. Singer, B.; Aslaksby, T.E.; Topalova-Casadiego, B.; Tveit, E.S. Investigation of Materials Used by Edvard Munch. Studies in Conservation 2010, 55, 274-292.

Page $17 / 24$ 
53. Colour index, 3d ed. ed.; The Society of Dyers and Colourists: Bradford, 1971.

54. MacEvoy, B. Handprint. Available online: https://www.handprint.com/HP/WCL/watery.html (accessed on 1/10/2022).

55. De Keijzer, M. The history of modern synthetic inorganic and organic artists' pigments, James \& James: London, United Kingdom, 2002; $\mathrm{p}$. 42.

56. News, P.R.A. Francisco Oller y sus tradicionales bodegones autóctonos. 2021.

\section{Figures}
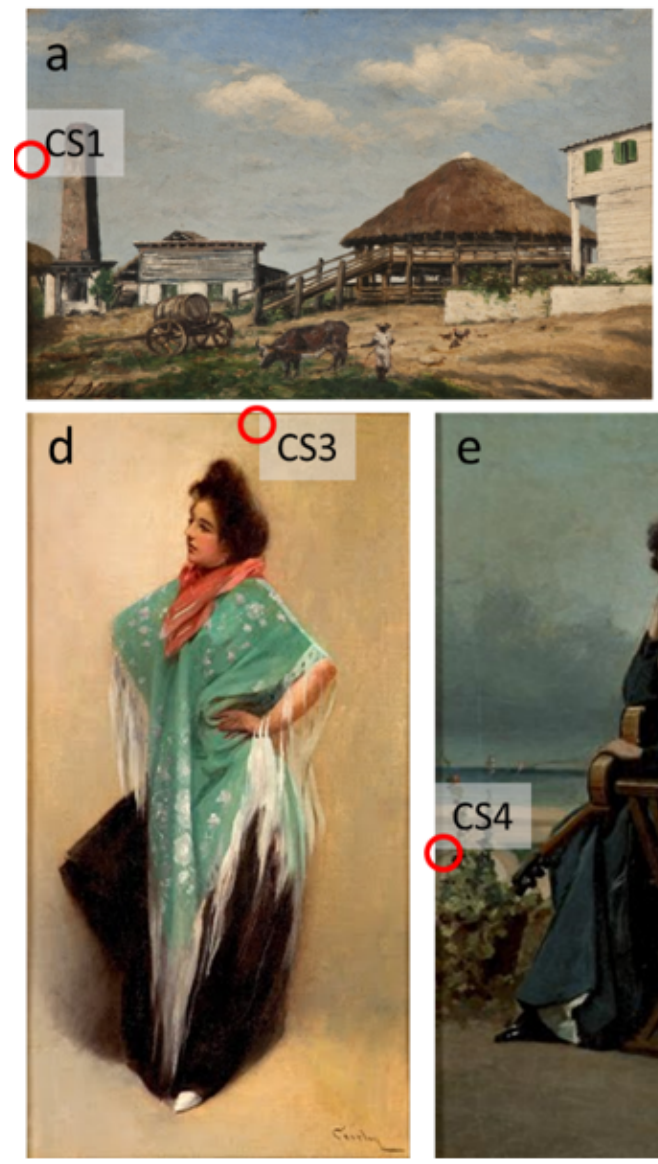
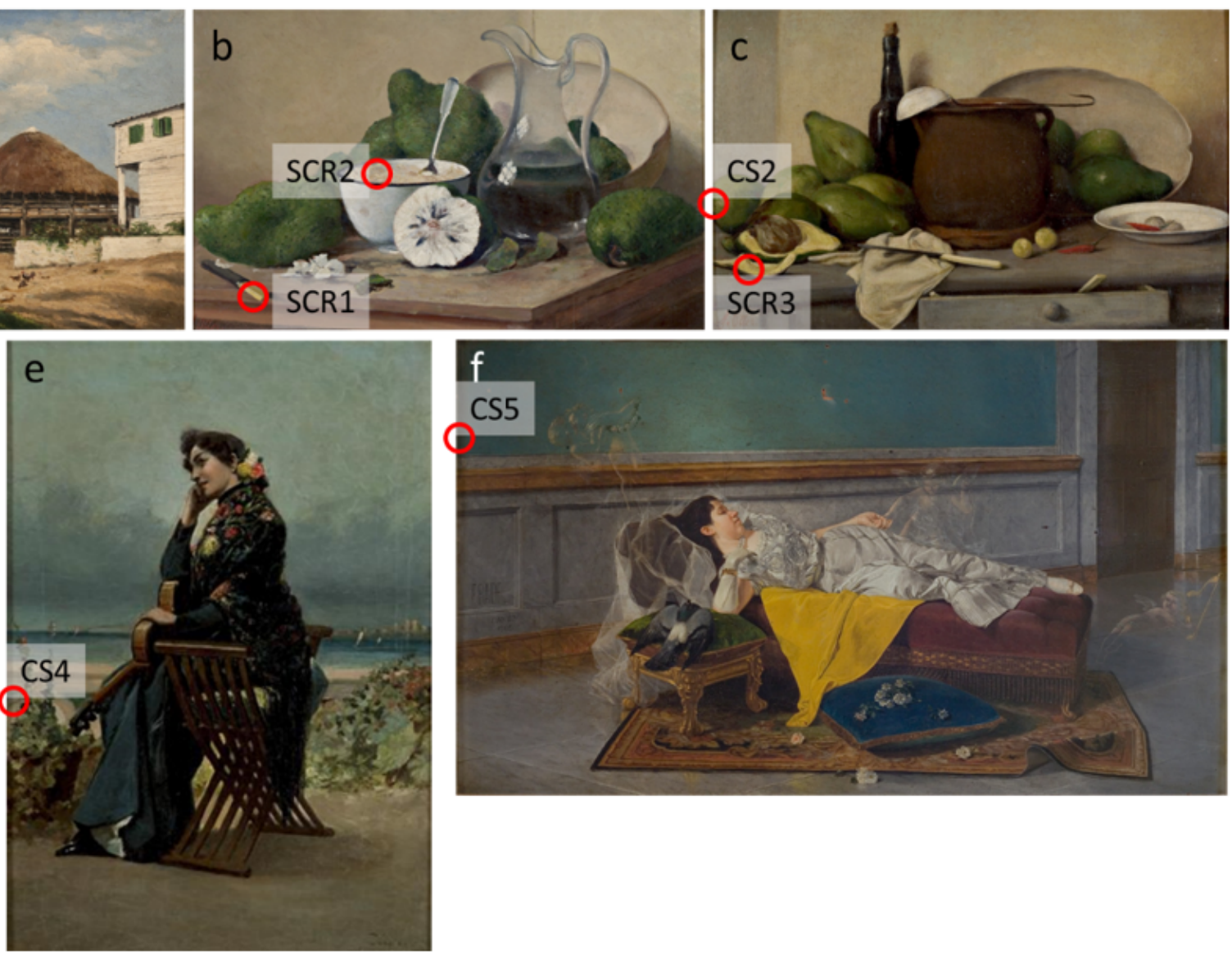

\section{Figure 1}

(a) "Trapiche Meladero" (1890, accession number: Trapiche), (b) "Bodegón con guanábanas” (1890, accession number: CAM-1329,), (c) “Bodegón con aguacates y utensilios" (1890-91, accession number: CAM-1328), all painted by Francisco Oller (1833 - 1917), (d) "La Chula” (1895, accession number: 2004-005), (e) “Mujer en la Playa” (1897, accession number: 2004-007), both painted by José Cuchí y Arnau (18571925), and (f) "Rêverie d'amour" (1905, accession number: 2003-05-02), painted by Ramón Frade. Sampling locations are indicated by the red circles and associated with the cross-section (CS) and/or scraping (SCR) numbers. 

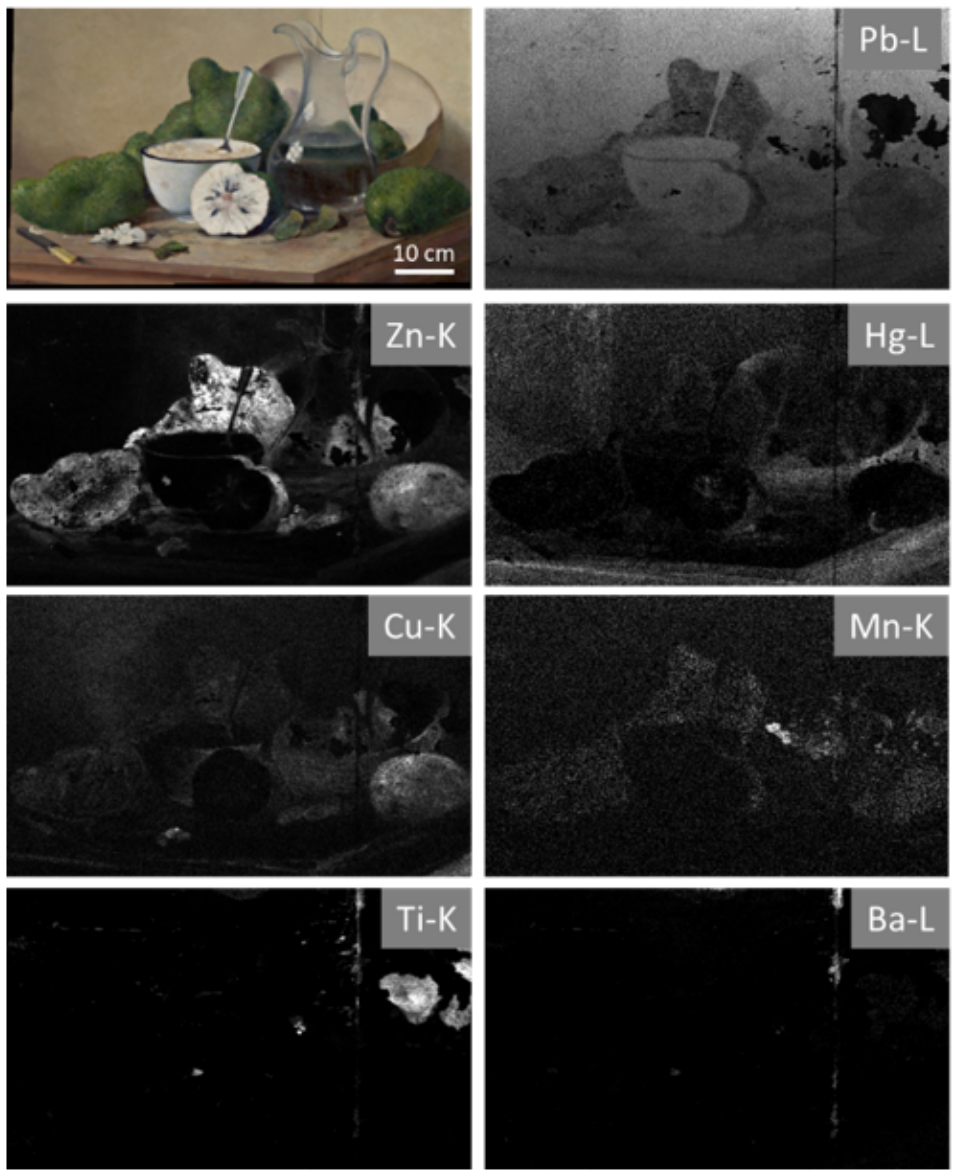
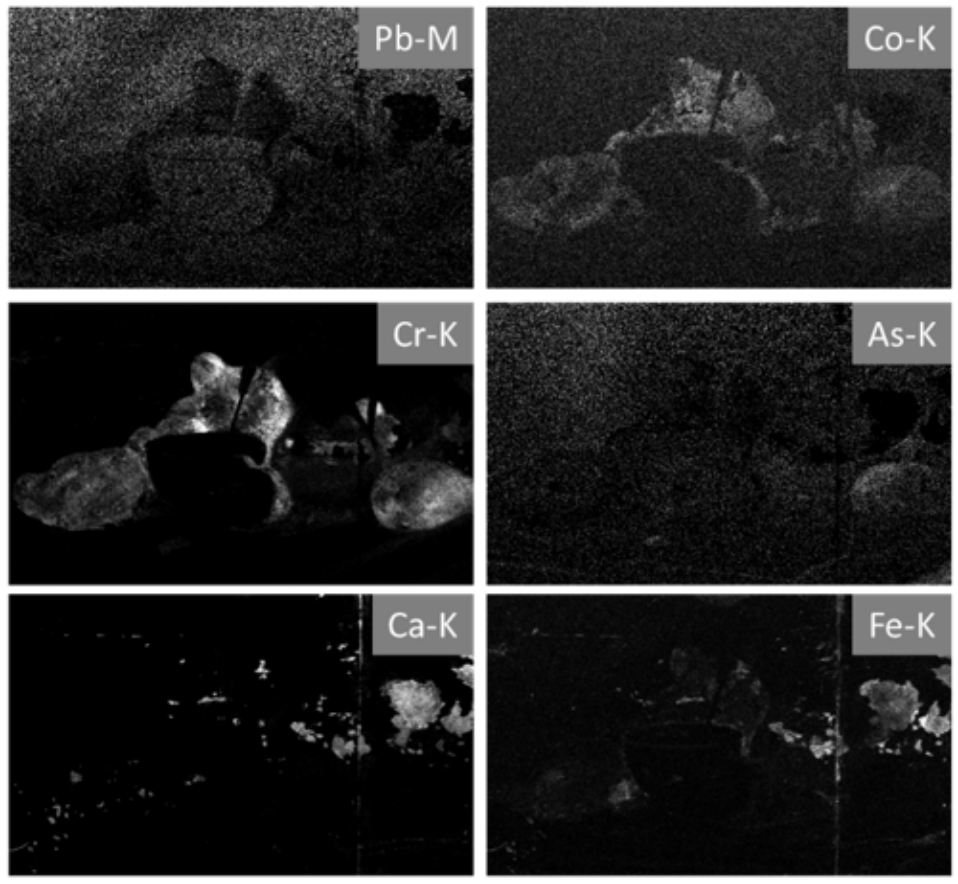

Figure 2

Registered RGB and MA-XRF elemental maps obtained for CAM-1329. Elemental maps for Ca, Fe, Ti, Ba and K appear to be associated with conservation intervention whereas $\mathrm{Pb}, \mathrm{Co}, \mathrm{Zn}, \mathrm{Hg}, \mathrm{Cr}, \mathrm{As}, \mathrm{Cu}, \mathrm{Mn}$ and some Fe appear associated with the original paint layer. Elemental distribution maps suggest the use of a lead-based ground (likely to be lead white); ochre-based retouching ( $\mathrm{Ti}$, K, Fe, Ca, K); cobalt blue/zinc white and/or cobalt green pigment (Co/Zn); pigment mixtures containing chrome-based green ( $\mathrm{Cr})$; vermilion $(\mathrm{Hg})$; Emerald/Scheele's green $(\mathrm{Cu} / \mathrm{As})$; and possibly umber pigment (Fe/Mn). 

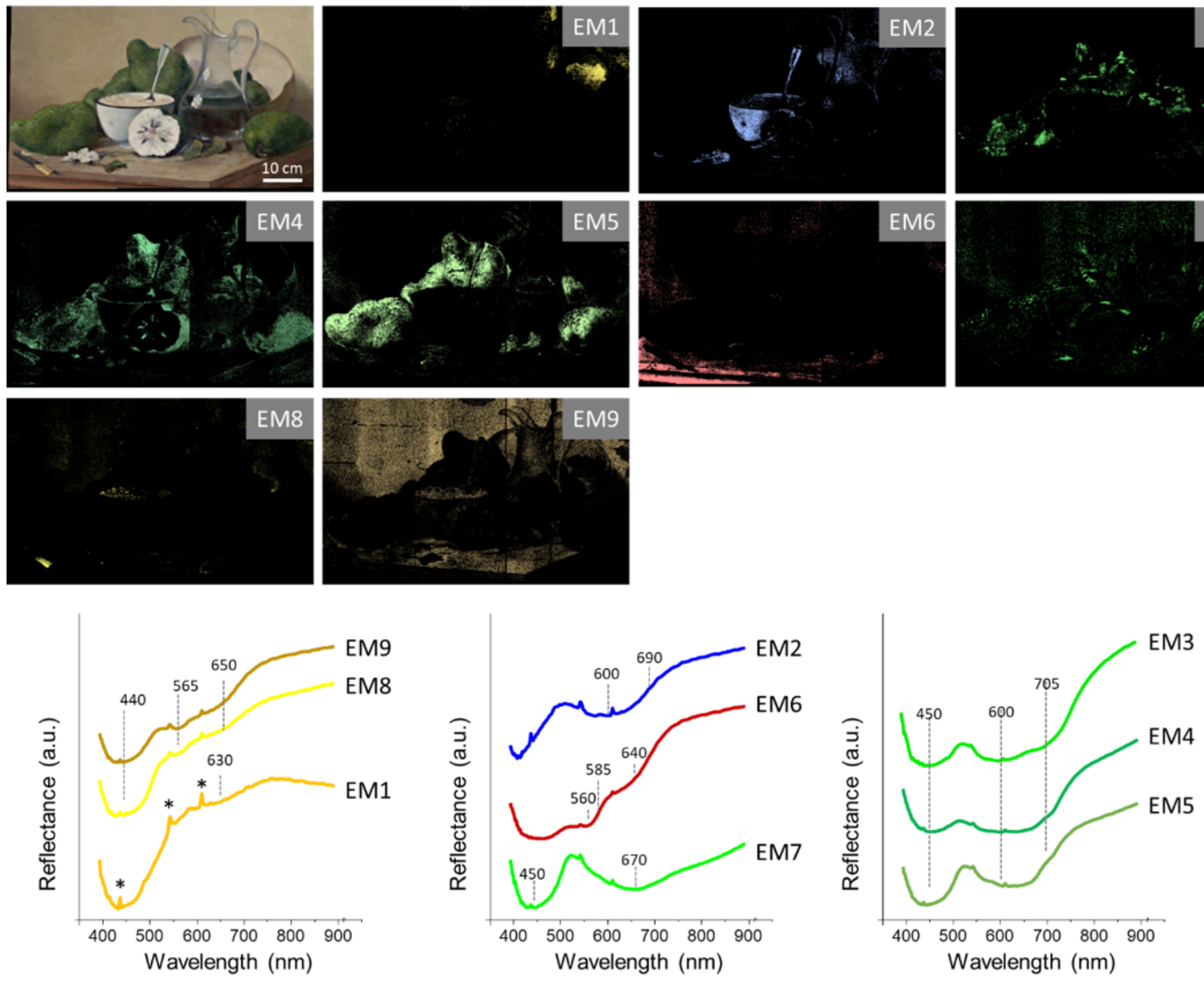

Figure 3

Registered RGB, RIS distribution maps and associated reflectance curves for each identified endmember (EM) in CAM1329. RIS distribution maps suggest the use of an ochre-based retouching (EM1); ultramarine blue (EM2); green pigment mixtures containing ultramarine blue (EM3, EM4 and EM5); vermilion and ochre mixture (EM6); a possibly Emerald/Scheele's green pigment (EM7); and unknown yellow pigments (EM8 and EM9). Sharp bands at 437, 543, and $610 \mathrm{~nm}$ (marked with an asterisk) are associated with light pollution from nearby ambient lightning.

a

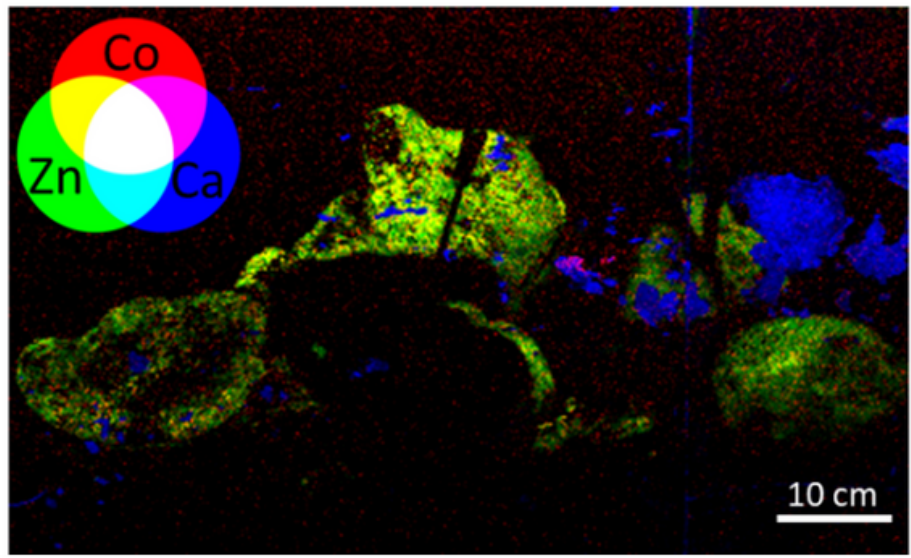

b

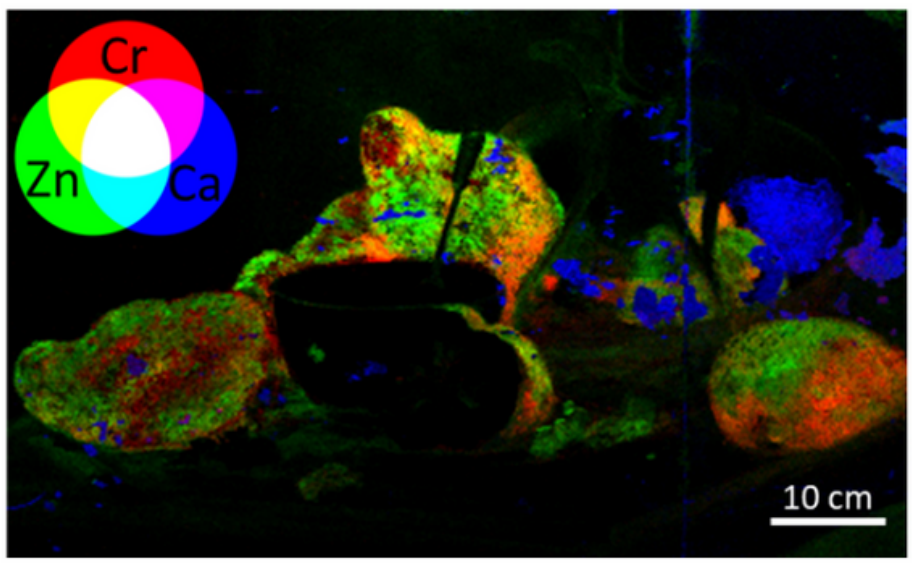

Figure 4 
RGB composite images for (a) Co (red), Zn (green) and $\mathrm{Ca}$ (blue) and (b) $\mathrm{Cr}$ (red), $\mathrm{Zn}$ (green) and $\mathrm{Ca}$ (blue) highlighting the elemental overlap of $\mathrm{Co}$ and $\mathrm{Zn}$ (yellow) rather than $\mathrm{Cr}$ and $\mathrm{Zn}$ in CAM1329. The nearly perfect Co/Zn overlap suggests the use of cobalt green and/or cobalt blue and zinc white mixture rather than the use of zinc chromate.
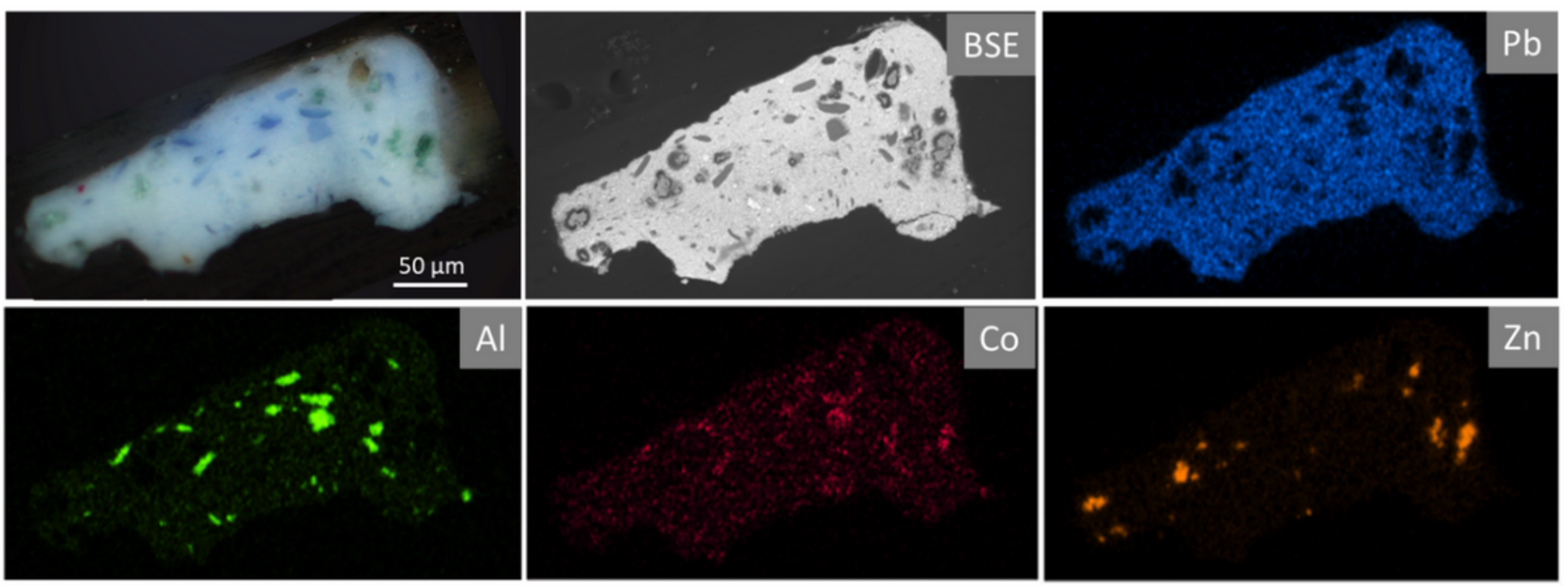

\section{Figure 5}

Visible light image of CS1, SEM backscattered electron image and Pb, Al, Co and $\mathrm{Zn}$ elemental maps highlighting the use of cobalt green $(\mathrm{Co} / \mathrm{Zn})$ along with cobalt blue (Co/Al) and lead white $(\mathrm{Pb})$ in the light blue sky of Oller's Trapiche Meladero.

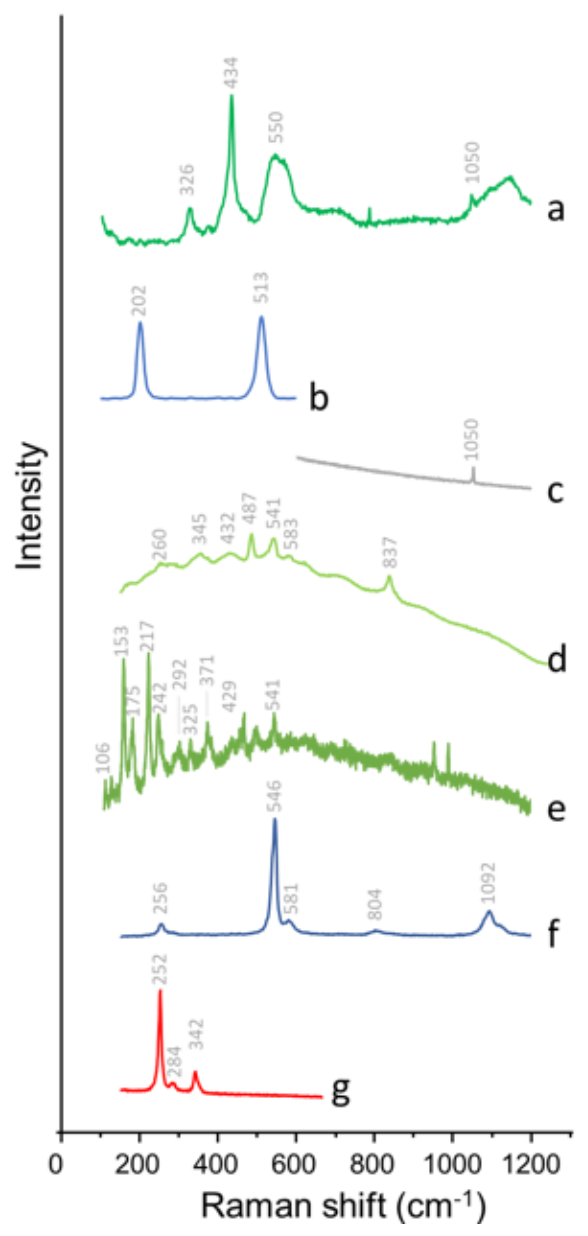

Figure 6 
Raman spectra of pigment particles found in CS1 and CS2. (a) cobalt green and lead white, (b) cobalt blue, all found in CS1; (c) lead white found in the ground of CS2, (d) viridian and (e) Emerald green, as two green pigments found in CS2; (f) ultramarine blue and (g) vermilion, both found in CS2.
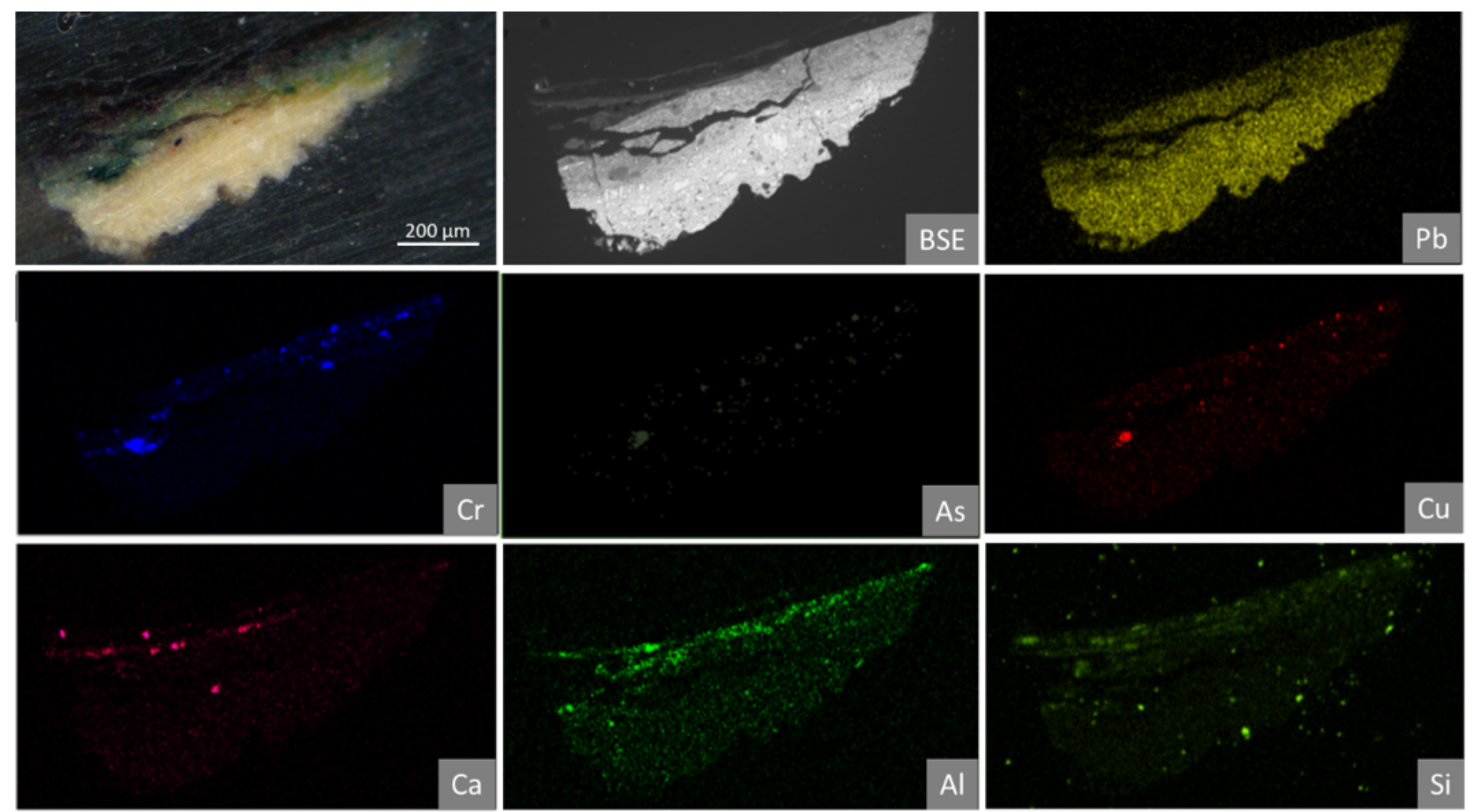

\section{Figure 7}

Visible light image, backscattered electron SEM image and EDX elemental distribution maps obtained on CS2. Images and SEM-EDX analyses suggest the presence of a lead white $(\mathrm{Pb})$ ground, followed by a $\mathrm{Cr}$ - and $\mathrm{Cu} / \mathrm{As}$-containing green layer. The presence of $\mathrm{Al}$ and $\mathrm{Si}$ in the upper layer also suggest the presence of ultramarine blue. The yellow areas of the sample did not yield any elemental response that would allow to identify the yellow pigment, which may suggest the use of an organic yellow pigment. 


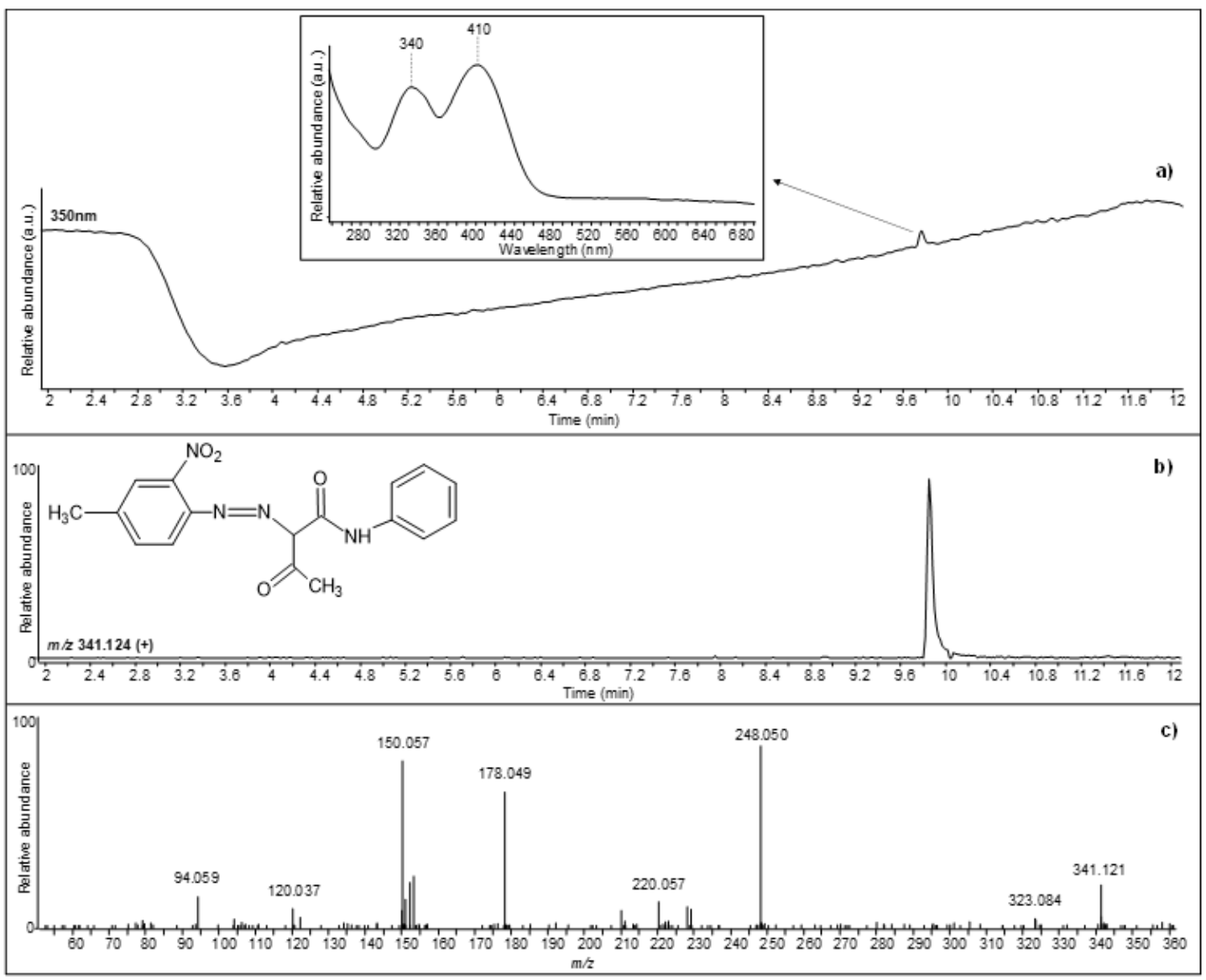

Figure 8

Chromatographic and mass spectrometric results obtained for sample SCR2 (painting CAM1329): a) UV-Vis chromatogram extracted at 350 $\mathrm{nm}$ and UV-Vis absorption spectrum (insert) of the compound detected at $9.8 \mathrm{~min}$ and identified as PY1 (Hansa Yellow G - C.I. 11680); b) extracted ion chromatogram obtained in positive ionization mode for $\mathrm{m} / \mathrm{z} 341.124$ corresponding to [M+H] ${ }^{+}$of $\mathrm{PY} 1$ (molecular structure of the neutral molecule also reported); c) corresponding tandem mass spectrum of $[\mathrm{M}+\mathrm{H}]^{+}$of PY1. 


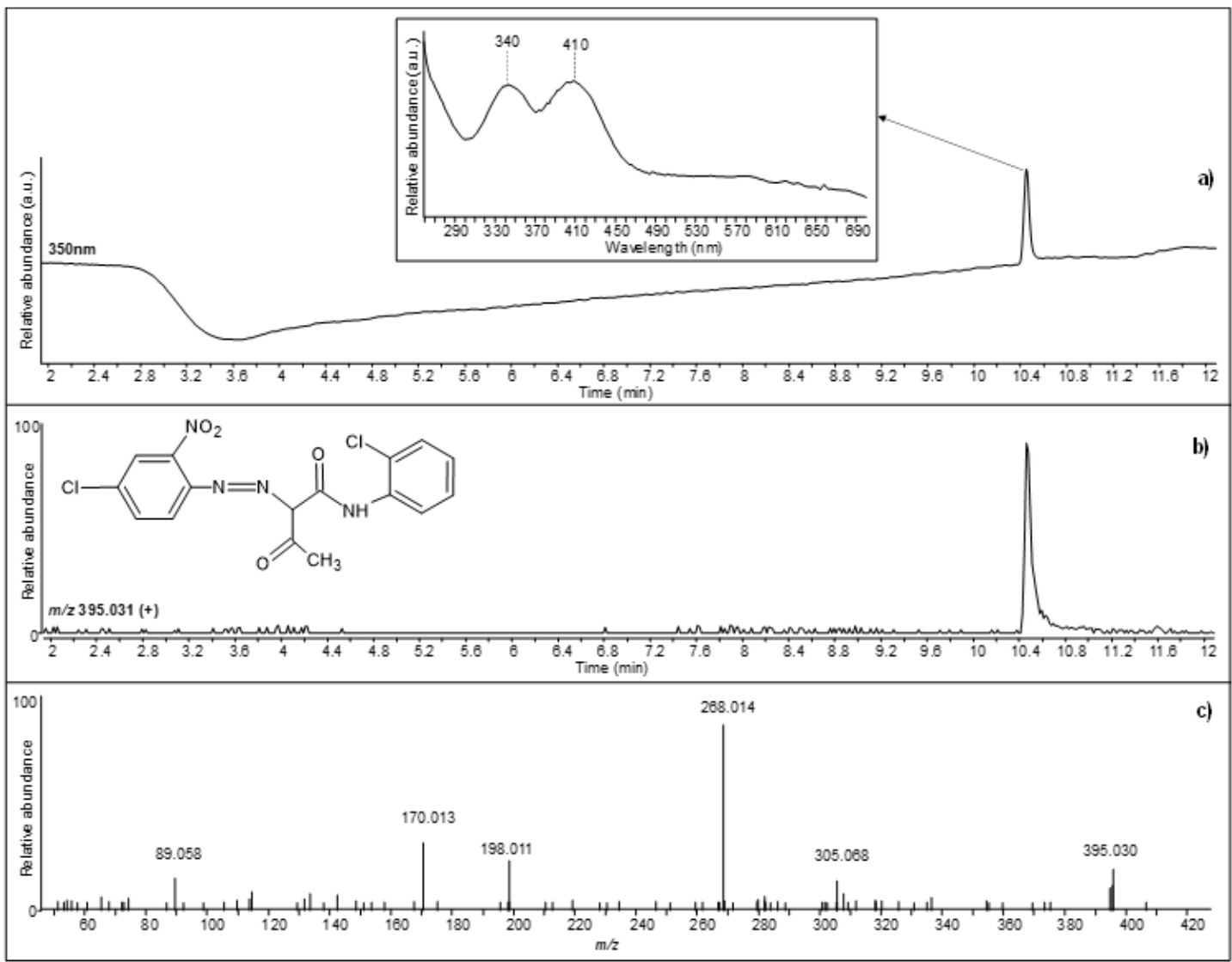

Figure 9

Chromatographic and mass spectrometric results obtained for sample SCR3 (painting CAM1328): a) UV-Vis chromatogram extracted at 350 $\mathrm{nm}$ and UV-Vis absorption spectrum (insert) of the compound detected at 10.4 min and identified as PY3 (Hansa Yellow 10G - C.I. 11710); b) extracted ion chromatogram obtained in positive ionization mode for $\mathrm{m} / \mathrm{z} 395.031$ corresponding to [M+H] ${ }^{+}$of $\mathrm{PY} 3$ (molecular structure of the neutral molecule also reported); c) corresponding tandem mass spectrum of $[\mathrm{M}+\mathrm{H}]^{+}$of $\mathrm{PY} 3$.

\section{Supplementary Files}

This is a list of supplementary files associated with this preprint. Click to download.

- SupplementaryInformation.pdf 